Florida International University FIU Digital Commons

FIU Electronic Theses and Dissertations

University Graduate School

11-15-2004

\title{
A study of gas holdup properties of selected non- Newtonian simulants
}

Milena Abarca

Florida International University

DOI: $10.25148 /$ etd.Fl13101500

Follow this and additional works at: https://digitalcommons.fiu.edu/etd

Part of the Mechanical Engineering Commons

\section{Recommended Citation}

Abarca, Milena, "A study of gas holdup properties of selected non-Newtonian simulants" (2004). FIU Electronic Theses and Dissertations. 1059.

https://digitalcommons.fiu.edu/etd/1059

This work is brought to you for free and open access by the University Graduate School at FIU Digital Commons. It has been accepted for inclusion in FIU Electronic Theses and Dissertations by an authorized administrator of FIU Digital Commons. For more information, please contact dcc@fiu.edu. 
FLORIDA INTERNATIONAL UNIVERSITY

Miami, Florida

\section{A STUDY OF GAS HOLDUP PROPERTIES OF SELECTED}

NON - NEWTONIAN SIMULANTS

A thesis submitted in partial fulfillment of the

requirements for the degree of

MASTER OF SCIENCE

in

MECHANICAL ENGINEERING

by

Milena Abarca 
To: Dean Vish Prasad

College of Engineering

This thesis, written by Milena Abarca, and entitled A Study of Gas Holdup Properties of Selected Non - Newtonian Simulants, having been approved in respect to style and intellectual content, is referred to you for judgment.

We have read this thesis and recommend that it be approved.

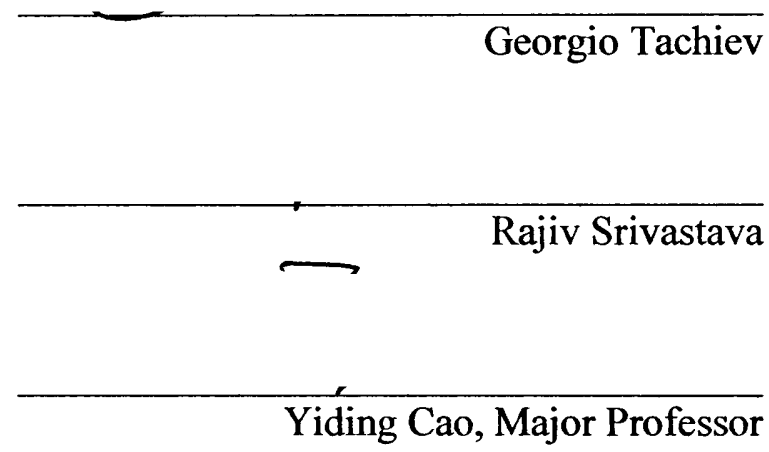

Date of Defense: November 15, 2004

The thesis of Milena Abarca is approved.

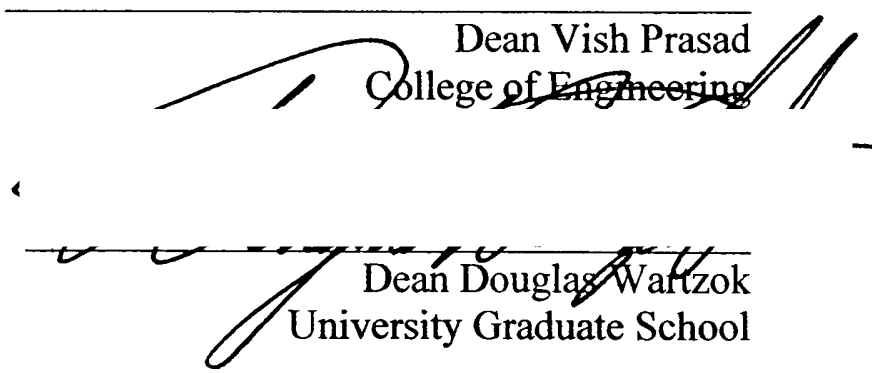

Florida International University, 2004 


\section{DEDICATION}

I dedicate this thesis to my husband Ignacio, to my parents Alberto and Yaneth, to my sisters Alejandra and Adriana, to my little brother Alberto Javier and especially to my uncle Fernando Berendique and my aunt Lissy Pacheco, who have encouraged and supported me during my studies. Without their support, the completion of this work would not have been possible. 


\section{ACKNOWLEDGMENTS}

I wish to thank my major professor, Dr. Yiding Cao, for making my dream come true to pursue my master's degree by providing guidance throughout my course of study. I would like to thank my advisor, Dr. Georgio Tachiev for his support, patience, tremendous encouragement during the thesis development and for conducting the experiments and data acquisition. My special thanks to Dr. Rajiv Srivastava for his continuous monitoring, guidance and comments on my thesis. I would like to express my gratitude to Amer Awaad for his unconditional help, support for conducting the experiments and data acquisition. 


\section{ABSTRACT OF THE THESIS \\ A STUDY OF GAS HOLDUP PROPERTIES OF SELECTED \\ NON - NEWTONIAN SIMULANTS \\ by \\ Milena Abarca \\ Florida International University, 2004 \\ Miami, Florida \\ Professor Yiding Cao, Major Professor}

The U.S. Department of Energy (DOE) needs a design basis to properly design a PJM and ventilation systems for the Waste Treatment Plant vessels. In order to meet DOE's needs for proper ventilation and PJM design technologies, Florida International University's Hemispheric Center for Environmental Technology (FIU-HCET) has studied the properties for gas holdup in selected non Newtonian fluids with physicochemical properties comparable to nuclear waste.

The primary purpose of this research was to study the holdup properties of selected non - Newtonian simulants and quantify the level of gas holdup in selected simulants using continuous argon injection in five gallons vessel. Gas holdup tests involved the injection of gas bubbles in simulant waste in scaled prototypic vessels. The holdup was measured as a function of injection rate in the vessel. Tests were performed with both Laponite, Clay 12\%, Clay 27\% and Qard 13.5. This work showed that the percentage of holdup was about $3 \%$ for all simulants despite the significant differences in rheology. 


\section{TABLE OF CONTENTS}

I.

INTRODUCTION

1.1. Scope and Purpose of this Study

II.

LITERATURE REVIEW 4

2.1. Classification of fluids 4

2.1.1. Definition of Newtonian Fluid 4

2.1.2. Non - Newtonian Fluid Behaviour 4

2.2. The Chemistry and Physics of Gas Retention and Release 6

2.2.1. Gas Generation 7

2.2.2. Gas Retention Mechanisms 8

2.2.2.1. Bubble Retention by Capillary Forces 9

2.2.3. Bubble Retention by Waste Strength 10

2.3. Gas Release 13

2.4. Bubble Formation 18

2.5. Single Bubble Analysis $\quad 20$

2.5.1. Static Model 20

2.5.2. Dynamic Model 23

2.6. Hydrogen Flammability Control Limit 30

2.7. Flammable Gas Theory 31

III. EXPERIMENTAL APPARATUS AND PROCEDURES

3.1. Experimental Scale Setup $\quad 34$

3.2. Test Apparatus for Gas Injection Experiments 36

3.2.1. Test Vessel 37

3.2.2. Mixer $\quad 37$

3.2.3. Pumps $\quad 39$

3.2.4. Headspace 40

3.3. Test Requirements for Gas Retention and Release Experiment 41

3.4. Test Procedures for Gas Retention and Release Experiments 41

3.4.1. System Assembly $\quad 42$

3.4.2. Vessel Filling 43

3.4.3. Gas Leak Test 44

3.4.4. Simulant Conditioning $\quad 45$

3.4.5. Steady State Holdup and Release Test 46

3.4.5.1. Gas Injection for Steady State Holdup 46

3.4.5.2. Gas Release from Steady State Holdup $\quad 47$

3.4.6. Predetermined Gas Holdup and Release Test 47

3.4.6.1. Gas Injection for Predetermined Holdup 47

3.4.6.2. Gas Release from Predetermined Hold-Up 49

3.4.7. Simulant Gelling and Gas Release $\quad 49$ 
3.4.7.1. Gas Release During Simulant Gelling 49

3.4.7.2. Gas Release from Gelled Simulant 50

3.4.8. Rheology Samples $\quad 51$

3.4.9. Steady State Tests 51

3.4.9.1. Release from Steady State Retention 51

3.4.9.2. Release from Gelled State Holdup $\quad 52$

3.5. Simulant Rheology 53

IV. EXPERIMENTAL RESULTS AND DISCUSSION 56

4.1. Experimental Observations $\quad 56$

4.1.1. Laponite Steady state Injection Test 56

4.1.2. Diluted Clay Steady State Injection Test 58

4.1.3. Clay $27 \%$ Steady State Release Test 60

4.1.4. QARD 13.5 Steady State Injection Test 63

4.1.5. QARD Steady State Release Test 65

4.2. Results Analysis $\quad 70$

V. CONCLUSIONS AND RECOMMENDATIONS 71

$\begin{array}{ll}\text { REFERENCES } & 73\end{array}$

$\begin{array}{ll}\text { APPENDICES } & 75\end{array}$ 
1. Rheology of Simulants Employed in Steady State Retention and Release Test

2. Rate of Release from $27 \%$ Clay during Steady State Release

3. Rate of Release from QARD 13.5 during Steady State Release

4. Comparison of Rate of Release from QARD 13.5 to Clay $27 \%$

5. Condition for the Static Mixer Scaling Test

6. Henry's law constant for solubility in water at $298 \mathrm{~K}$

7. Henry's law constant for solubility in water at $298 \mathrm{~K}$

8. Henry's law constant for solubility in water at $298 \mathrm{~K}$

$\begin{array}{lll}\text { 9. Blue Laponite Shipping Details and Properties } & 85\end{array}$

10. Clay Simulant Shipping Details and Properties 86

11. 24\% Clay Simulant Shipping Details and Properties 87

12. QARD Sludge Simulant Shipping Details and Properties 88

13. Calibrations Standards and Certificate of Conformance for Argon 91

14. Statistical Value for Argon Concentration $=0 \mathrm{ppm} \quad 89$

15. Statistical Value for Argon Concentration $=84.2 \mathrm{ppm} 93$

16. Statistical Value for Argon Concentration $=1501 \mathrm{ppm} 95$

17. Statistical Value for Argon Concentration $=5013 \mathrm{ppm} \quad 97$

18. Statistical Value for Argon Concentration $=99600 \mathrm{ppm} \quad 99$ 


\section{LIST OF FIGURES}

FIGURE

PAGE

1. Different Types of Fluids Behaviour

2. Bubble Diameter Vs. Yield Stress (Pa)

3. Relative bubble size with respect to LDC diameter

4. Relatively size of bubble compare to LDC diameter obtained by the static and dynamic method

5. Experimental Setup for Study Gas Retention and Release Behavior of Non-Newtonian Simulants

6. Test Apparatus Showing Test Vessel with Mixer Rake Modification

7. Gas bubbles dispersion in Laponite simulant

8. Shear Stress - Shear Rate Behaviour of Simulants before Testing

9. Shear Stress - Shear Rate behaviour of Simulants after Testing

10. Argon Concentration During Release from Steady State with Laponite Simulant

11. Argon Concentration during Release from Steady - State Retention with Clay Simulant

12. Gas Holdup Detected in the diluted Clay Simulant after steady State Injection Test

13. Argon Concentration during Release from Steady State Retention with $27 \%$ Clay Simulant

14. Rate of Release from $27 \%$ Clay during Steady State Release

15. Argon Concentration during Injection into QARD 13.5 until reaching Steady State Condition

16. Gas Holdup Detected in the diluted QARD Simulant after Steady State Injection Test 
17. Argon Concentration during Release into QARD 13.5 until reaching steady state condition

18. Rate of Release from QARD 13.5 during Steady State Release

19. Rate of Release Comparison Curve for QARD and Clay

20. Schematic Test Set up for Static Mixer Scaling Test

21. Logic Diagram for Static Mixer Scaling Test

22. Calibration Graph for Argon Concentration $=0 \mathrm{ppm}$

23. Calibration Graph for Argon Concentration $=82.4 \mathrm{ppm}$

92

24. Calibration Graph for Argon Concentration $=1501 \mathrm{ppm}$

94

25. Calibration Graph for Argon Concentration $=5013 \mathrm{ppm}$

96

26. Calibration Graph for Argon Concentration $=99600 \mathrm{ppm}$

98

27. Calibration Curve for Argon; Concentration Range from 84.2 to 99600 ppm 


\section{LIST OF SYMBOLS}

\begin{tabular}{cl} 
WTP & Waste Treatment Plant \\
LFL & Lower Flammability Limit \\
PJM & Pulse jet Mixer \\
DOE & Department of Energy \\
LEL & Lower Explosion Limit \\
$N_{B o}$ & Bond Number \\
$H_{S}$ & Height of lithostatic column above bubble \\
$D_{p}$ & Mean pore diameter \\
$\Delta \rho$ & Difference between solid and liquid density \\
$\sigma$ & Surface Tension \\
$\tau_{y}$ & Yield stress \\
A & Area ratio related to how yield stress resists \\
& bubble expansion \\
$\Delta h$ & Maximum height \\
$\rho_{L}$ & Liquid density \\
$g$ & Acceleration of gravity \\
$D_{b}$ & Bubble diameter \\
$\rho_{w}$ & Bulk waste density \\
$\alpha_{N B}$ & Gas fraction \\
$F_{b}$ & Buoyancy force \\
$\rho_{\mathrm{s}}$ & Sludge bulk density \\
$\tau_{0}$ & Gas density \\
& Critical force \\
& Dimensionless yield constant \\
& Sludge yield stress \\
\hline & \\
\hline &
\end{tabular}


Hydrogen gas generation by radiolysis and/or thermolysis has been recognized as a significant hazard within the waste treatment plant (WTP) ${ }^{1}$. During normal operation, pulse jet mixers ${ }^{2}$ in the WTP vessels with non-Newtonian waste must achieve safe, controllable release of flammable gas, hydrogen in particular. This requires: a) waste mobilization throughout the vessel and b) demonstration of acceptable gas holdup levels at steady state operation. The current control strategy is to maintain hydrogen concentrations below $25 \%$ of the lower flammability limit (LFL). The lower flammability limit has been defined as $2.5 \%$ of hydrogen concentration in the gas space. The design approach involves providing sufficient dilution ventilation during all plant conditions (e.g. normal operating and upset conditions) and therefore requires an accurate understanding of hydrogen generation rate within each WTP vessel.

Despite the large number of studies that have been performed, the understanding of the process of gas generation, gas release and gas holdup within nuclear waste material is still inadequate. Because of the complex nature of the fluid, difficulties in conducting experiments with real waste, and the vast number of variables involved in

' Waste Treatment Plant (WTP) at Hanford/ORP, which is a nuclear waste storage and treatment facility area located in Washington state owned and operated by the Department of Energy

${ }^{2}$ Pulse Jet Mixers (PJM) will be employed for mixing the "black cells" at WTP 
the process, current knowledge in this field is mainly empirical and involves simulants mostly.

\subsection{SCOPE AND PURPOSE OF THIS STUDY}

The U.S. Department of Energy (DOE) needs a design basis to properly design a PJM and ventilation systems for the Waste Treatment Plant vessels. In order to meet DOE's needs for proper ventilation and PJM design technologies, Florida International University's Hemispheric Center for Environmental Technology (FIU-HCET) has studied the properties for gas holdup in selected non Newtonian fluids with similar physicochemical properties comparable to nuclear waste. Gas holdup tests involve the injection of gas bubbles in simulant waste in scaled prototypic vessels. The holdup (fraction of gas retained in simulant) was measured as a function of injection rate in the vessel. Tests were performed with both Laponite (required for visualizing holdup behavior) and representative particulate simulants which included Clay $12 \%$, Clay $27 \%$ and QARD 13.5.

The primary purpose of this research is to study the holdup and rate of release properties of selected non-Newtonian simulants with different rheology. The selection of the simulants was made based upon two principle modes of operations:

- Normal operation where all expected utilities are available and the primary concern is achieving and maintaining good blending of various high solid slurries. In this case, the tank slurries are always maintained in a fluid state so that the yield stress and perhaps the viscosity (consistency) are the key parameters. 
- Design Basis Events where the primary air and/or other utilities may be lost and emergency utilities are used to maintain the process vessels in a safe state. The primary issue of concern in this case is to maintain the gas space in the process tanks at a hydrogen gas concentration that is less than the lower explosion limit (LEL) using a minimum of utilities. The yield strength and yield stress and to some lesser extent the viscosity (consistency) of the slurry will need to be considered.

The significance of the study is that it will provide critical experimental data which will support the efforts of WTP to design the PJM and ventilation systems for a range of operating conditions including normal operation as well as emergency design based events (up to $96 \mathrm{hrs}$ power failure). This work will also provide a better understanding of the gas holdup properties of non-Newtonian simulants and the results will be applicable to other engineering areas including: non-Newtonian fluid and slurries processing, tank mixing applications requiring solid mixing, solid suspension and fluid blending. 


\subsection{CLASIFICATION OF FLUIDS}

The fluids can be classified as Newtonian and non - Newtonian fluids due to the effect produce under the action of shear stress.

\subsubsection{DEFINITION OF A NEWTONIAN FLUID}

A Newtonian fluid is fluid that has constant viscosity at all shear rates at a constant temperature and pressure and can be described by one parameter rheological model. An equation describing a Newtonian fluid is given below

$$
\tau=\mu \gamma
$$

where $\tau$ is the shear stress, $\mu$ is the fluid viscosity and $\gamma$ is the shear rate.

\subsubsection{NON - NEWTONIAN FLUID BEHAVIOUR}

A non - Newtonian fluid is one whose flow curve (shear stress versus shear rate) is non linear and do not pass through the origin, for example where the apparent viscosity, shear stress divided by shear rate, is not constant at a given temperature and pressure but it is dependant on flow conditions such as flow geometry, shear rate, etc. and sometimes even on kinematics history of the fluid element under consideration. Such material may be conveniently group in three general classes: 
1. fluids for which the rate of shear at any point is determined only by the value of shear stress at that point at that instant; these fluids are variously known as time dependant, purely viscous, inelastic or Newtonian fluid.

2. more complex fluids for which the relation between shear stress and shear rate depends, in addition, upon the duration of shearing and their kinematic history, they are called time dependant fluids, and finally,

3. substances exhibiting characteristics of both ideal fluids and elastics solids and showing partial elastic recovery, after deformation; these are categorized as viscoelastics fluids.

Equation 1 implies that the shear rate value at any point within the sheared fluid is determined only by the current value of shear stress at that point or vice versa. Depending upon the form of the function of the equation 1 , these fluids may be further subdivided into three types: pseudoplastic, viscoplastic or dilatant. Qualitative flow curves on linear scales for these three types of fluid behaviour are shown in figure 1 , the linear relation typical of Newtonian fluids is also included.

It is of particular interest the non - Newtonian fluids that have viscoplastic fluid behaviour, especially bingham fluids. Since the rheology of the simulants used in this research study resembles this type of behaviour. This type of fluid behaviour is characterized by the existence of a yield stress $\left(\tau_{0}\right)$ which must be exceeded before the fluid will deform or flow. Conversely, such material will deform elastically when the extremely applied stress is smaller than the yield stress. Once the magnitude of the external stress has exceeded the value of the yield stress, the flow curve must be linear or 
non linear but will not pass through the origin. A fluid with a linear flow curve is called bingham plastic fluid and is characterized by a constant plastic viscosity (the slope of the shear stress versus shear rate curve) and a yield stress. It is interesting to note that viscoplastic materials also display an apparent viscosity which decreases with increasing shear rate.

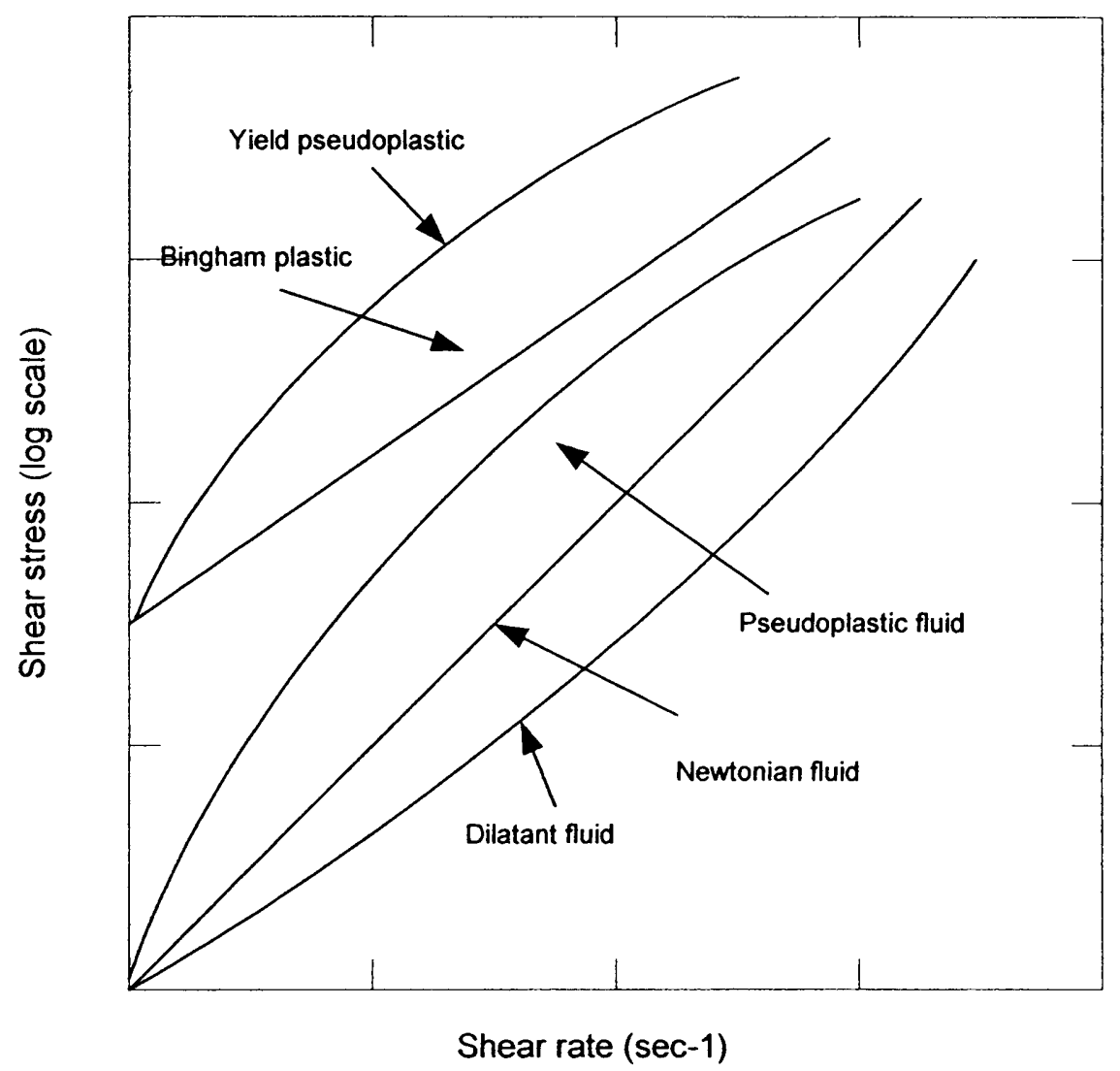

Figure 1 Different types of fluids behaviour

\subsection{THE CHEMESTRY AND PHYSICS OF GAS RETENTION AND RELEASE}

Understanding gas generation phenomena and predicting the gas release rate as well as gas retention rate within waste treatment plant vessels are important in controlling 
the flammable gas hazard and planning operational activities in the WTP vessels. There are two main approaches for studying gas generation, gas release and gas retention phenomena: experimental and theoretical. In this chapter, the theoretical and experimental work from previous research in this field is discussed with the main emphasis on recent developments.

\subsubsection{GAS GENERATION}

Hydrogen in the Nuclear Waste Tanks is mostly generated by the following:

- Radiolysis of water

- Corrosion of the steel tank walls

- Cascade during radiolytic and chemical oxidation reactions of organic compounds in the waste

Previous studies have shown that the rates of gas generation are really sensitive to temperature and depend on the concentrations of radioactive isotopes. Hu (2000) developed an empirical rate equation that describes the dependence of the hydrogen generation rates on the properties of the radiolytic constituents and chemical content of the tank waste and its temperature. Comparison of the calculated generation rates and the observed release rates indicated that the calculated generation rates were within a factor of 2 to 3 of the field observations 


\subsubsection{GAS RETENTION MECHANISMS}

Hydrogen generated within the waste is not very soluble within the wastes and comes out of the waste as bubbles. Bubbles percentage is the most important mode of gas retention and release, concerning flammability. The principal methods of gas retention can be divided in three groups:

- Bubbles retained by direct attachment to particles

- Bubbles retained between particles by capillary forces

- Bubbles retained by the strength of the surrounding waste

In layers of liquid saturated settled solids particles, bubbles retention is dominated by capillary forces and the waste strength; direct attachment of bubbles to particles plays a minor role. The increased waste strength that might result from future decreases in waste temperatures is not expected to increase gas retention (G. Johnson 2001).

There are five mechanisms of bubble retention P.A. Gauglitz (1994):

- Viscous retention occurs when the fluid's yield strength, which is the stress when plastics deformation occurs, is sufficient to hold the bubbles. Bubbles larger than a critical value will overcome the yield strength of the fluid and rise.

- Agglomerates of bubbles and particles are held together.

- When particles accumulate at the bubble interface and protect the bubble from coalescing with other bubbles these types of bubbles are known as Armored bubbles. 
- Capillary forces retained bubbles when the bubbles can not go through the narrow openings between the particles.

- Bubble attachment refers to a bubble attached to a particle.

\subsubsection{BUBBLE RETENTION BY CAPILLARY FORCES}

Bubbles can be held in the interstitial spaces or pores between particles by capillary forces when the lithostatic load of the waste is sufficient to hold the particles in contact against the force of the bubble's internal pressure trying to push them apart. This retention mechanism requires either relatively large pores, which reduces the internal bubble's pressure, or a deep waste column, which increases the lithostatic load or both. These bubbles assume an irregular, dendritic shape conforming to the passages between the particles. When the internal pressure of a bubble overcomes the effect of lithostatic load, it pushes the surrounding particles apart. The bubble is then restrained by yield strength of the bulk waste as a particle - displacing bubble. Whether a bubble is held by yield strength or capillary force is indicated by the Bond number. This dimensionless number contains two parameters, a ratio of gravitational force to surface tension force, and a ratio of waste strength force to surface tension force. If the number exceeds unity, a bubble is held by capillary forces between particles in the pore - filling configuration (G. Johnson 2001). The Bond Number can be express as follows:

$$
N_{B_{o}}=\frac{\Delta \rho g H_{s} D_{p}}{4 \sigma}+\frac{\tau_{Y} D_{P}}{4 \sigma} A
$$


Where $\mathrm{g}$ is the acceleration due to gravity, $\mathrm{H}_{\mathrm{s}}$ is the height of the lithostatic column above the bubble, $D_{p}$ is the mean pore diameter through which a bubble must pass to escape retention. Assume to be represented by the particle diameter, $\Delta \rho$ is the difference between solid and liquid density, $\sigma$ is the surface tension, $\tau_{\mathrm{Y}}$ is the yield stress, $\mathrm{A}$ is an area ratio related to how the yield stress resists bubble expansion; it was experimentally estimated to be 2.8 (Gauglitz 1995).

When the bubble internal pressure overcome the restraining force of surface tension, gas pushes out of the top of the bubble and liquid flows into bottom of the bubble causing the bubble to move upward. The gas volume fraction at which this motion occurs is called the percolation threshold. The maximum height, $\Delta \mathrm{h}$, that a pore - filling bubble can attain before percolating is expressed as (G. Johnson 2001)

$$
\Delta h=4 \sigma / \rho_{L} g D_{P}
$$

Where $\sigma$ is the surface tension, $\rho_{\mathrm{L}}$ is the liquid density; $\mathrm{g}$ is the acceleration of the gravity, $D_{p}$ the mean pore diameter through which a bubble must pass to escape retention. It is assumed to be represented by the particle diameter.

\subsubsection{BUBBLE RETENTION BY WASTE STRENGTH}

If a bubble is retained, the bubble shape will be determined by a balance of surface tension and waste strength. With relatively weak waste or small bubbles, surface tension pulls bubbles into an approximately spherical shape. If the effect of waste strength is greater than surface tension forces, the bubbles grow into the weakest area of the waste 
surrounding it and assume dendritic shape. The maximum diameter of round bubbles, $D_{b}$, before they begin to assume a dendritic shape is given by (G. Johnson 2001):

$$
D_{b}<\frac{\sigma}{\tau_{y}}
$$

Where $\tau_{\mathrm{y}}$ is the yield stress, $\sigma$ is the surface tension, $\mathrm{D}_{\mathrm{b}}$ is the bubble diameter. A bubble can grow vertically until its buoyancy exceeds the ability of the waste to hold it in place. The release of small bubbles in this manner is believed to comprise the steady state release observed in waste tanks (G. Johnson). A criterion for incipient motion of a solid sphere immersed in a Bingham fluid can be derived in terms of yield stress, bulk density and a critical gravity yield number, $\mathrm{Y}_{\mathrm{G}}$ (Chabbra and Uhler 1986). The resulting limiting diameter is expressed as

$$
D_{b}<\frac{\tau_{y}}{\rho_{w} g Y_{G}}
$$

Where $\rho_{\mathrm{w}}$ is the bulk waste density, $\mathrm{g}$ is the acceleration of gravity, $\mathrm{Y}_{\mathrm{G}}$ is estimated to be 0.2 (Stewart et al. 1996a). The combination criteria of equations 3 and 4 limited the round bubble diameter to 0.5 to $1 \mathrm{~cm}$. This size is essentially constant over the range of Hanford tank waste properties (bulk non-convective layer density of 1500 to $1800 \mathrm{~kg} / \mathrm{m}^{3}$, surface tension of 0.08 to $0.1 \mathrm{~N} / \mathrm{m}$ ) as shown in figure 1 . The range of yield stresses shown on the abscissa represents the expected range of tank waste strength. This is consistent with observations of bubbles in waste samples and simulants (G. Johnson 2001). 
Equation 4 along with figure 1 gives the limiting height of dendritic, particle displacing bubbles. When they grow to the limiting height, the increased hydrostatic pressure difference pinches off the bottom of the bubble and push the top of the bubble upward in a manner similar to the migration of pore - filling bubbles. However, the motion of particle displacing bubbles in much faster since it does not require liquid through flow through a porous medium. This percolation of dendritic, particle displacing bubbles is believed to be the primary mechanism for the spontaneous release observed in the head space gas monitoring data. Figure 1 indicates that the limiting height of dendritic bubble increases with waste strength. Waste with a yield stress of $3500 \mathrm{~Pa}$ could theoretically support a dendritic region $1 \mathrm{~m}$ in height. However, experiments have shown that if the waste yield stress exceed a few hundred Pascals, the bubble do not collapse and a connected network forms that allows the gas to escape and prevent further retention (Gauglitz et al 1996). 


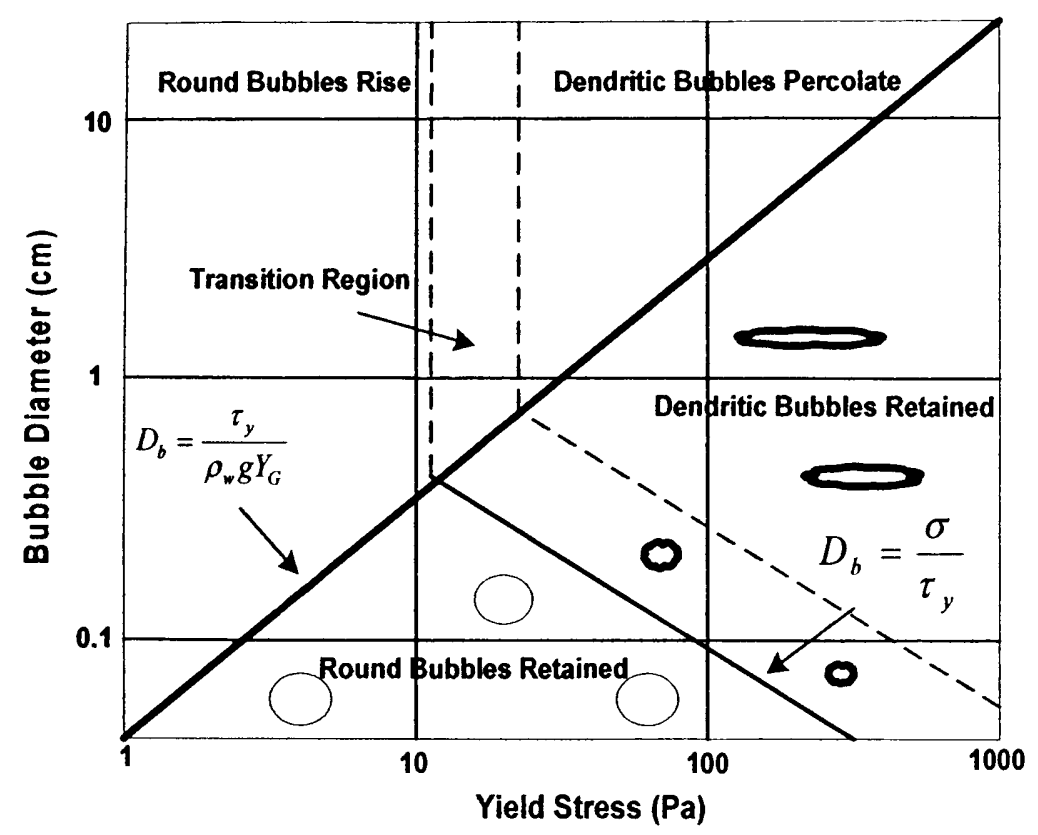

Figure 2 Bubble diameter Vs. Yield Stress (Pa). (Gauglitz et al 1996)

\subsection{GAS RELEASE}

Gas release mechanisms are determined by the waste composition and physical properties. The effects of the different wastes types and configuration on retention and released are considered and described as follows:

Waste Types and Configurations

There are three types of waste that can be classified as follows:

- A solution of dissolved inorganic and organic compounds in water that contains at most a small fraction of suspended solid particles. Liquid that lies over a layer of settled solids is called supernatant. 
- Sludge consists of fine particles that are mostly metal oxides

- Salt cake is the settled precipitate of sodium salts crated from sodium saturated solution. Salt cake is composed of particles that are generally larger than the sludge.

Waste configuration is the arrangement of waste types in layers within a tank. The waste types are distributed within the tanks in only a few different configurations, depending mainly on the amount of liquid that can be described as follows:

- Liquid which is almost entirely liquid waste type

- Liquid over sediment where a deep layer of supernatant overlies an equally deep layer of which liquid is saturated sediment

- Solid over liquid which is the entire mass of undissolved solids in the tanks floats on a dense liquid layer

- Wet sediment which is a sediment saturated with liquid, and there is little or no supernatant

- Pumped which has had interstitial liquid reduced by saltwell pumping such that the interstitial liquid level is below the waste surface

- Crust a solid over a liquid arrangement consisting of a relatively thin floating layer of gas bearing solids

- Mixed slurry in which most of the solid particles and small gas bubbles are kept in suspension mechanically 
Gas releases in this waste configuration result from the sediment layer becoming buoyant with respect to the supernatant and are called buoyant displacement gas release events (G. Jonhson 2001).

Gas Release by Buoyant Displacement

A buoyant displacement gas release is originated in tanks with a deep layer of supernatant when a portion of the settled solids accumulates sufficient gas to become buoyant with respect to the liquid above it, breaks away and rinses through the liquid. The stored gas bubbles expand as the portion rinses, disrupting the surrounding waste so portion of the gas can escape into the dome space. The portion sinks back to the bottom of the tank after releasing gas is not longer buoyant. The gas fraction, $\alpha_{\mathrm{NB}}$, required for the sediment layer to become neutrally buoyant is defined by (G. Johnson 2001):

$$
\alpha_{N B}=1-\frac{\rho_{L}}{\rho_{W}}
$$

Where $\rho_{\mathrm{L}}$ is the liquid density and $\rho_{\mathrm{W}}$ is the sediment bulk gas - less density.

The accumulation of gas until it reaches buoyancy depends on the balance between steady - state gas release and gas generation. If generation exceeds release, gas will accumulate. In general, the release rate increases as gas accumulates so that it eventually balanced generation, and equilibrium is achieved. 
There are several others ways of gas release that can be mentioned depending on the case. Some of those examples can be mentioned as follows:

- Mechanical disturbances such as saltwell pumping used in west sediment configurations single shell tanks

- Gas release caused by seismic events is a natural phenomena form of release

- Background or steady state release that usually occurs continuously by both bubble detachment and evaporation at varying rates. Generally is proportional to the gas generation rate in the waste. If the gas generation rate is very high the background release rates are very low and are diluted to insignificance by passive ventilation.

- Induced releases are caused by a disturbance to the waste such as mixing, saltwell pumping, retrieval and waste transfer.

- Spontaneous release events have been observed but are typically insignificant, representing only temporary elevations in the background gas release rate. It is important to mention that only Buoyant Displacement Gas Release Events (BDGREs) that occur in some double shell tanks (DSTs) have resulted in hydrogen concentrations exceeding 25\% of the LFL (Mahoney 2002).

Buoyant Displacement Gas Release Events

Bubbles trapped in the sediment grow until sediment becomes buoyant with respect to the liquid above. At that moment large portions of sediments break free and rise to the surface, where they disintegrate and release a fraction of the gas to the headspace. These 
releases are relatively rapid and have produced the highest headspace hydrogen concentration (Mahoney 2002). Evaporative ammonia during (BDGREs) is small to negligible despite the temporary surface agitation during the event. Most of the ammonia is released with the bubbles, and the measured hydrogen/ammonia ratios in the headspace during BDGREs are similar to those of retained gas.

Induced Gas Releases

Several types of disturbances can produce significant bubble releases, including mixing pump operation and liquid retrieval can also induce BDGREs that are also dominated by bubble releases, including mixer pump operation and saltwell pumping. These operations can also induce BDGREs that are also dominated by bubble release. Except saltwell pumping these disturbances do not produce significance concurrent evaporation of ammonia. Mixing mostly affects the waste near the tank bottom and does not appear to disturb the surface sufficiently to significantly enhance evaporation (Mahoney et al. 2002).

Evaporative releases favor liquid surfaces and high concentration of dissolved ammonia in the liquid. If a liquid surface is stirred, the evaporative mass transfer rate can greatly increased. Transferring liquid waste into a tank is the primary waste disturbance that creates liquid surface to increase evaporation.

The maximum ammonia concentration in the headspace resulting from an evaporative release corresponds to a partial pressure equal to the equilibrium vapor pressure of the dissolved ammonia. Saltwell pumping causes slow releases from both 
bubble and evaporation. Evaporation occurs from unsaturated but still moist, porous waste remaining after the drainable interstitial liquid has been removed. Some bubbles are removed promptly through expansion when the liquid above them is removed and the hydrostatic pressure reduced. Others are not release until liquids drains from around them.

Background Gas Release

In tanks where waste is not influenced by waste disturbing operations or undergoing a gas release event, which is defined as a rise of more than $100 \mathrm{ppm}$ in the headspace hydrogen concentration followed by an exponential decay. The less soluble gases are release at the same rate they are generated except in some cases where a small fraction remains trapped in the waste as bubbles. This is reflected by a gradual rise in waste level or interstitial liquid level. Since ammonia is so soluble approximately $99 \%$ of the ammonia generated remains in solution in the waste rather than being release to the headspace. Background release is only a potential concern when the hypothetical minimum ventilation rate produced by barometric pressure fluctuations, because this may exceed the $25 \%$ LFL.

\subsection{BUBBLE FORMATION}

G.Terrones (2002) proposed two models of bubble formation within the sludge based on the assumption that all gas originates from a single point source. In this analysis static and dynamics effects are considered, as well as spherical configuration of the bubbles during formation and detachment stages. 
Several experimental observations have been done in determining the force that keeps a buoyant sphere motionless. In this static model the value of drag force that is representative from the experimental observations has been used. In the dynamic model, the effect of gas generation rate is incorporated into the analysis. This model is based on the generalization of a model for bubble formation and detachment developed and validated for Newtonian fluids by Kumar and Kuloor (1970), G. Terrones(2002).

Sludge plug formation scenarios: there are four patterns of bubble formation in the sludge. First, bubbles are originated from multiple point generation sources of gas within sludge of low yield stress, in this case small bubbles will grow and move upward overcoming the low yield stress of the material. Second, bubbles are originated from multiples sources of gas within the sludge of high yield stress; in this case bubbles will grow but will remain motionless for a longer period of time before they move upward overcoming the high yield stress of the sludge. In this case it is possible the coalescence with neighboring bubbles forming larger bubbles that could span the diameter of the containing vessel. Third, a single bubble could grow as a result of a large concentration of uranium metal particles in small region of the vessel. In this particular case bubble growth can be viewed as being dominated by a single point source, which depending on the mechanical properties of the sludge and the gas generation rate could lead to a vessel spanning bubble. Fourth, a layer of gas could grow due to a uniformly distributed concentration of gas generating particles over an area equal to the cross - sectional area of the containing vessel. 


\subsection{SINGLE BUBBLE ANALYSIS}

\subsubsection{STATIC MODEL}

In the static model of bubble formation, the bubble size is determined by a force balance between buoyancy and the drag force exerted on the bubble by the sludge yield stress. Surface tension is neglected. In reality, large bubbles deviate considerably from perfect spheres because the interfacial force at the bubble boundary is small compared to other forces. Yet it is assumed that the bubble remains spherical (G. Terrones 2002).

A value of drag force has been used that is thought to be representative for all this experimental results (Atapattu et al. 1995), since a relatively small number of experimental results are available for a Non- Newtonian flow compare to that of Newtonian flow..

For spherical bubbles the buoyancy force $(\mathrm{Fb})$ can be defined as follows:

$$
F_{b}=\frac{\pi}{6}\left(\rho-\rho_{s}\right) g d^{3}
$$

Where $\rho$ is the sludge bulk density, $\rho$ s is the gas density (hydrogen in this case), $g$ is the gravitational acceleration, $\mathrm{d}$ is the bubble diameter. 
Adapting Apattu's (1995) experimentally determined condition of no motion to a bubble retained in sludge, the total critical force $\mathrm{Fc}$ which a bubble will overcome the yield stress and move upward is

$$
F_{c}=\frac{\pi d^{3} \tau_{0}}{6 k}
$$

Where $\mathrm{K}$ is dimensionless yield constant and $\tau 0$ is the sludge yield stress.

There is not universal value for $\mathrm{k}$, the $\mathrm{k}$ value depends on the rheological properties of the medium. The usual range is from 0.04 to 0.6 .

To reduce the number of varying parameters that determine whether a bubble can rise through the sludge under static conditions, a dimensionless number can be obtained from a ratio between the critical force and the buoyancy force on the bubble. This leads to a dimensionless parameter, Ns, given by

$$
N_{s}=\frac{\tau_{0}}{k\left(\rho-\rho_{g}\right) g d}
$$


A bubble overcomes the yield stress of the sludge if $\mathrm{Ns}>1$ and the condition at the onset of motion requires that $\mathrm{Ns}=1$. To compare the bubble size relative to the diameter of the LDC at the onset motion, the following relationship is used

$$
\frac{d}{D}=\frac{\tau_{0}}{k\left(\rho-\rho_{s}\right) g D}
$$

where D is the diameter of the LDC. Plotting equation (3) where the ordinate and the abscissa are the left and right side of the above mentioned equation will allow us to see a representation of a relative bubble size that would allow us to determine the likelihood of encountering a vessel-spanning bubble within the LDC containing sludge of known density and yield stress. Since the density of the gas is at least three orders of magnitude smaller than that of the sludge, the dimensionless number that will be needed to calculate the relative size of the bubble can be defined as: ${ }^{\tau} /(k \rho g D)$. In this model it is assume that a bubble will continue to grow, until it either becomes buoyant or spans the diameter of the LDC. From the static bubble formation, a vessel spanning bubble will occur for sludge yield stress in excess of 1000Pa. 


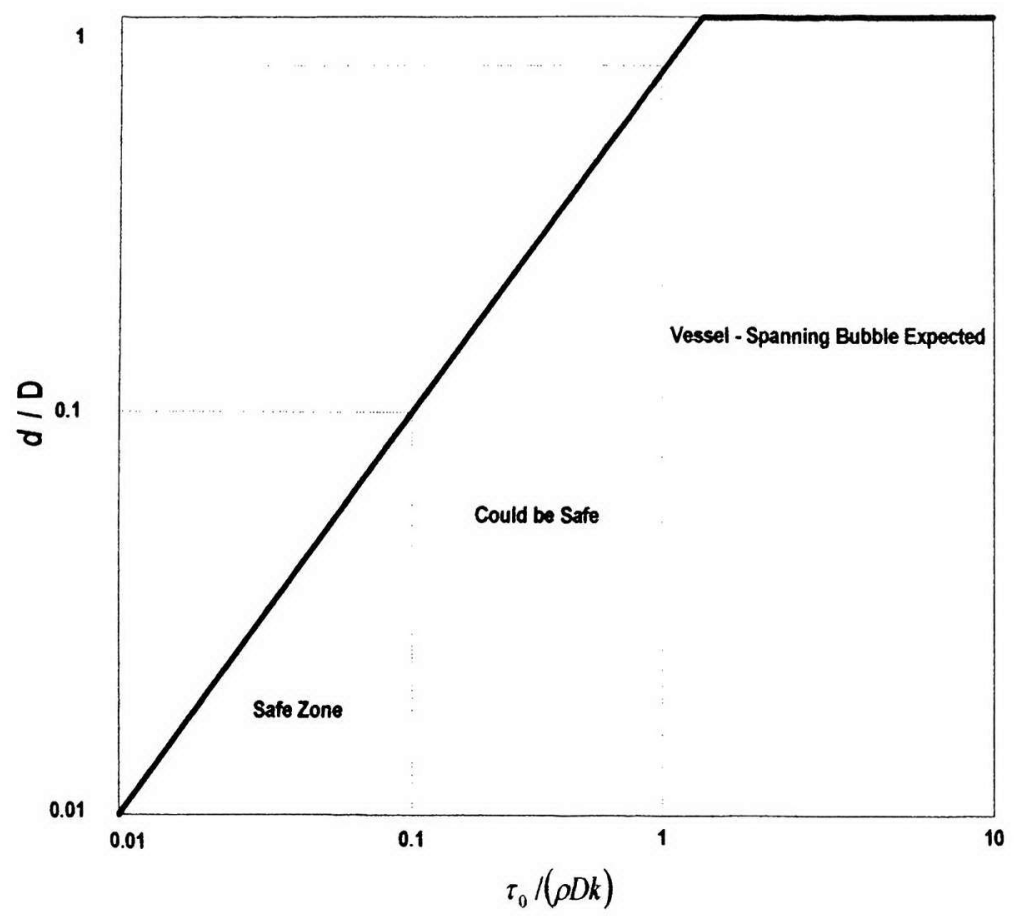

Figure 3. Relative bubble size with respect to LDC diameter.

\subsubsection{DYNAMIC MODEL}

This model was developed to determine how the gas generation rates influences the size of the bubble being formed within the sludge. This model will help us determine how much larger a bubble could grow as a result of a constant gas generation rate. In this model the assumption of constant rate of gas generation is conservative as the assumption made before that the bubble will remain spherical throughout.

This model is based on an initial expansion stage followed by a detachment stage. During the first stage the bubbles grow from a fixed point source of gas in such a way that base of the bubble remains at rest. The second one begins when the bubble starts moving upward while still connected to the gas source throughout a thin conduit, in this 
stage the bubble continue to grow prior a final detachment. The actual detachment process of a bubble from a source begins with spherical bubbles become increasingly elongated in the vertical direction as it grows. At the end of the first stage, the buoyancy and opposing forces are in equilibrium. During the detachment stage, buoyancy dominates and bubbles begin to rise. One of the model assumptions is the detachment time $\mathrm{T}$ takes place when the bubble base during the second stage has traversed a length equal to the radius of the bubble volume V1 reached at the end of the first stage. The final volume of the bubble V2 can be expressed as follows:

$$
V_{2}=V_{1}+Q T
$$

where $\mathrm{Q}$ is the gas generation rate in $\left(\mathrm{m}^{\wedge} 3 / \mathrm{s}\right)$, and $\mathrm{V} 1$ is obtained from the equation (8)

$$
V_{1}=\frac{\pi}{6}\left[\frac{\tau_{0}}{k\left(\rho-\rho_{g}\right) g}\right]^{3}
$$


In the detachment process the bubbles will experience two opposing forces: 1) drag due to the bubble vertical displacement and 2) the reaction force that the fluid exerts as a result of the rate of change of momentum due to expansion. There is a force associated with the expansion of a bubble even in the absence of viscosity, the net effect of which is calculated from the virtual mass, M, of the bubble (Kumar and Kuloor 1970), and can be expressed as follows:

$$
M=\frac{11 \rho V(t)}{16}
$$

Where $\mathrm{V}(\mathrm{t})$ is the instantaneous bubble volume at time $\mathrm{t}$ ( measured after the end of the first stage). At any time $t$, the bubble radius $r(t)$ is

$$
r(t)=\left[\frac{3\left(V_{1}+Q t\right)}{4 \pi}\right]^{1 / 3}
$$

The velocity at the center of the mass, $U_{c m}(t)$, is the sum of the velocity of the bubble at the base, $u(t)$, and the velocity due to the rate of expansion, $\operatorname{dr}(\mathrm{t}) / \mathrm{dt}$. Equation 13 can be transformed to:

$$
\frac{d\left(M u_{c m}\right)}{d t}=\rho g\left(V_{1}+Q t\right)-F_{D}
$$


Where $F_{D}$ is the drag force for the sphere moving in a viscoplastic medium. From Atapattu's (1995) and G. Terrones (2002) experimental correlation of the drag coefficient of sphere in a Herschel-Bulkley fluid, the drag force is

$$
F_{D}=12 \pi r^{2}\left[a \tau_{0}+X(n) \eta\left(\frac{u}{d}\right)^{n}\right]
$$

where a is a constant of order one that depends on rheology of the material and for which several values have been postulated, $\eta$ is the consistency factor, $\mathrm{n}$ is the behavior index, and X(n) is the drag correction factor computed by Gu and Tanner(1985). Their results an be fitted into the following function:

$$
X(n)=\frac{c_{1}+c_{2} n+c_{3} n^{2}+c_{4} n^{3}}{1+c_{5} n+c_{6} n^{2}+c_{7} n^{3}}
$$

with the corresponding coefficient values of

\begin{tabular}{|l|l|}
\hline Constant & Value \\
\hline$C_{1}$ & 1.290728601 \\
\hline$C_{2}$ & -0.66416093 \\
\hline$C_{3}$ & 13.94828967 \\
\hline$C_{4}$ & -10.8519716 \\
\hline$C_{5}$ & -0.87641226 \\
\hline$C_{6}$ & 9.308756042 \\
\hline$C_{7}$ & -5.71381689 \\
\hline
\end{tabular}


This has a correlation coefficient of 0.99968 . The condition of the onset detachment leads to the following equation from which the constant a can be evaluated in terms of all the known parameters

$$
a=\frac{1}{18 k}-\frac{X(n) \eta}{\tau_{0}}\left(\frac{\rho g k}{\tau_{0}}\right)^{3 n}\left(\frac{Q}{\pi}\right)^{n}-\frac{11}{144 \pi^{2}}\left(\frac{\rho g k}{\tau_{0}}\right)^{4} \frac{\rho Q^{2}}{\tau_{0}}
$$

Trying to simplify the above equation and introducing the following dimensionless expression

$$
N_{1}=\frac{\tau_{0}}{X(n) \eta}\left[\frac{\pi}{Q}\left(\frac{\tau_{0}}{\rho g k}\right)^{3}\right]^{n}
$$

The equation (11) can be written as follows:

$$
a=\frac{1}{18 k}-\frac{1}{N_{1}}
$$


Simplifying accordingly these simplifications lead to a first - order nonlinear differential equation, where $\mathrm{N}=\mathrm{N}_{1} / 18 \mathrm{k}$. Applying the corresponding transformations we get $\gamma$ that is the ratio between the bubble volume at the end of the detachment stage and the bubble volume obtained from purely static conditions. This will lead us to a nonlinear equation that will be solved numerically for $\gamma$. The value of length scale of the bubble under dynamic conditions is determined by the following equation:

$$
\left(\frac{d}{D}\right)_{\text {Dynamics }}=\gamma(N)\left(\frac{d}{D}\right)_{\text {Static }}
$$




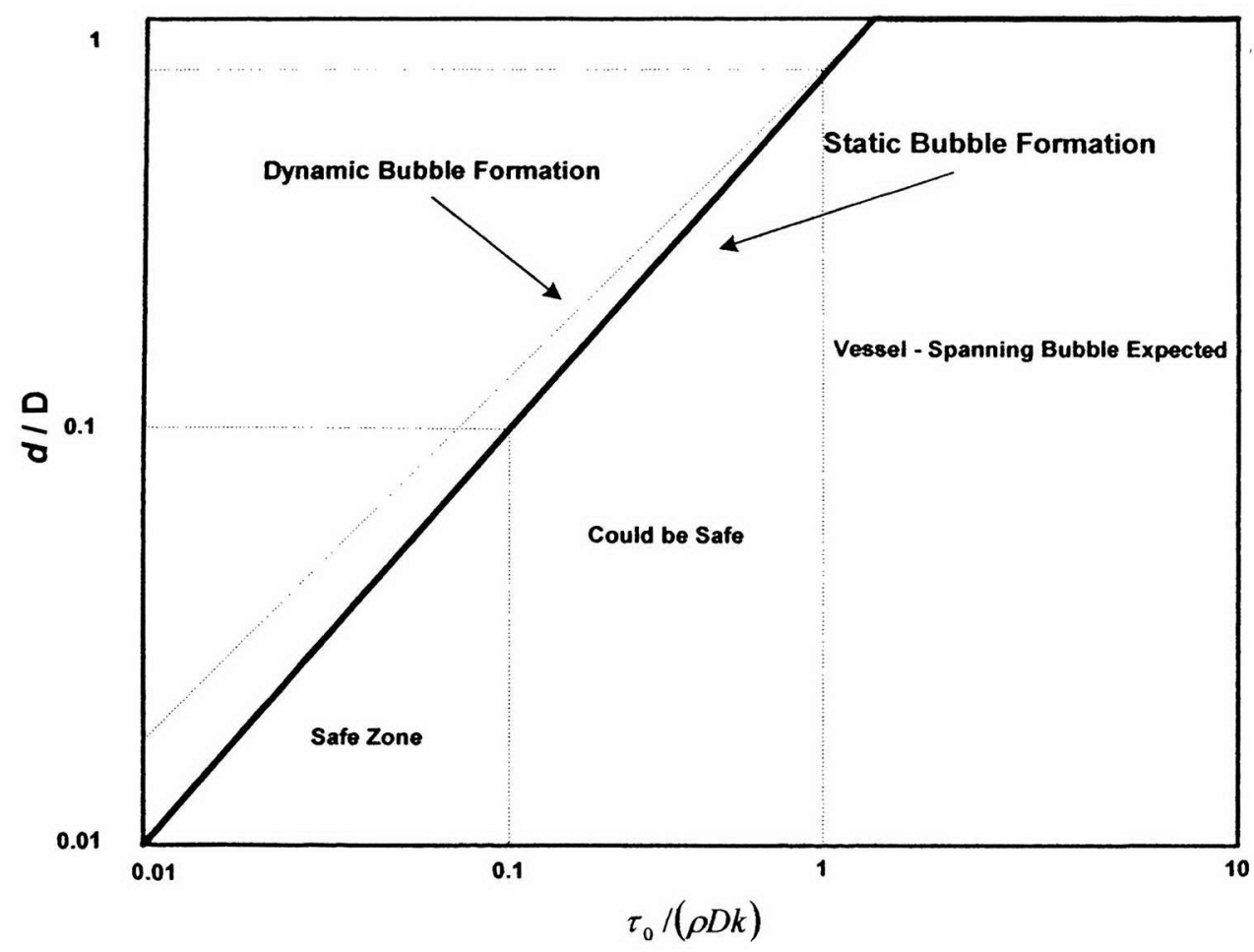

Figure 4 Relatively size of bubble compare to LDC diameter obtained by the static and dynamic method (G. Terrones 2002)

In Figure 3 there is a comparison between the relatively bubbles diameter calculated based on the static and dynamic models of bubble formation. The effect of gas generation rate in the dynamic model produces a larger - diameter bubble than its static counterpart, as shown in the figure 3. This effect is more pronounced in sludge with low yield stress. As the yield stress increases, the dynamics model calculations asymptotically approach those based on the static model. From the point of view of dynamic bubble formation, a vessel spanning bubble will occur for sludge yield stress values in excess of $800 \mathrm{~Pa}$. 


\subsection{HYDROGEN FLAMMABILITY CONTROL LIMIT}

The most relevant flammable gas generated in the WTP is hydrogen. However, there are additional fuel gases of potential concern, such as ammonia, methane and other hydrocarbons.

The flammability of a gas mixture depends on the concentration of each of the component gases with its own flammability limit as a pure gas. Mahoney (2002) says that extensive measurements and laboratory testing have shown that only hydrogen and ammonia contribute significantly to the flammability of the gases generated, retained and released by WTP.

There are three types of gas release: 1) Gas mixture generated, is directly released into the headspace of the tank where it is diluted by ventilation. 2) Some others retain a significance volume of gas and periodically release some of the stored gas into the tank headspace 3) Gas release when gelling after power failure.

There are several operations such as waste transfer and retrieval, saltwell pumping, etc. that disturb the waste and induce gas releases. These operations must be controlled to prevent ignition of flammable gas concentration in tanks headspace or other volume that could potentially release radioactive or toxic material.

There are potentially hazardous scenarios that need to be controlled to mitigate the hazard: (1) Accumulation of steady state or background gas releases in poorly ventilated tank headspaces, pit, risers and connected air spaces, and waste intrusive equipment (2) 
Release of gas accumulated within the waste matrix by natural spontaneous mechanisms such as buoyant instability or induced by waste disturbance.

For security purposes a LFL of $25 \%$ has been adopted. A hydrogen concentration of $6,250 \mathrm{ppm}$ is specified to represent $25 \%$ of hydrogen concentration of a mixture containing $2.5 \%$ vol hydrogen and $2 \%$ vol ammonia. These mixture would be just flammable (100\% LFL) on the conservative assumptions that LFLs for pure hydrogen and ammonia were, respectively, 3.5 vol\%(35,000 ppm) and 8 vol\% $(80,000 \mathrm{ppm})$ (Mahony 2002).

The control limit of $6,250 \mathrm{ppm}$ is recommended and is quite conservative since pure hydrogen in air has an LFL of 4 vol\% (40,000 ppm), and pure ammonia in air has an LFL of $15 \%$ vol $(150,000 \mathrm{ppm})$. It has been recommended that a control limit of 7,500, 8,000 or $9,000 \mathrm{ppm}$ hydrogen could properly represent $25 \%$ of the LFL.

In this summary an examination of the existing theory, data and experience with flammability measurements in Hanford radioactive waste tanks to evaluate the technical for the existing control limits and to determine weather any change is warranted is to be presented.

\subsection{FLAMMABLE GAS THEORY}

Several gas mixtures containing hydrogen, nitrogen, nitrous oxide, ammonia, methane, and other organic compounds are encountered in the headspaces of WTP and in the liquid and solid waste they contain. 
In particular, hydrogen is formed in WTP in three distinct ways: from radiolysis of water, during the cascade of radiolytic and chemical oxidation reactions of the organic compounds, and during the corrosion of the steel tank walls (Mahoney 2002).

In the process of gas generation the corrosion contribution is negligible and the organic decomposition portion is greater than that of water radiolysis. Ammonia arises from the oxidation reactions of the nitrogen containing complexants and by the hydrolysis of nitriles and amides that are produce during the oxidation of organic compounds. Nitrogen and nitrous oxide, as well as methane and other hydrocarbons, are produce along with ammonia during the complex degradation sequences of the complexants and other organics.

Due to the fact that most flammable gases are not soluble in the waste they come out of the solution to form discrete bubbles. In liquid waste, the bubbles rise to the surface and deposit their gas into the headspace. Bubbles that are formed in the liquid solid sediment are held by surrounding particles and continue to grow in place, where the gas in the bubbles assumes a composition determined by the equilibrium vapor pressure of each gas as its exists in solution.

The LFL of a dilute combustible fuel mixture is the smallest concentration that just supports flame propagation when stimulated by an external ignition source. The LFL is determined by the gas composition, energy and location of the ignition source; direction of flame propagation relative to gravity; and whether the gas is quiescent or being mixed. 
The gas composition effects include those associated with different oxidants and those associated with inert constitutes (Mahoney 2002). 
In this chapter experimental apparatus and the procedure for the bench scale gas holdup test system is described. This system is used to determine the gas holdup properties for selected non-Newtonian fluids such as slurries of kaoline, laponite which have different fluid parameters (viscosity and shear strength) and particle morphology and sizes.

These experiments were run by Georgio Tachiev and Amer Awaad at HCET facilities. The purpose of this research was mainly to perform the data analysis.

\subsection{EXPERIMENTAL SCALE SETUP}

The use of pulse jet mixers inside the vessels is a very intermittent process by its nature. Moreover, the fluid flow pattern is dependant on different factors such as the number of pulse tunes, and their distribution and fluid discharge geometries within the vessel. As an alternative a well - mixed vessel with a mechanical agitator was selected to represent the bounding case of a well - mixed vessel using PJM's. The typical configuration of pulse jet mixers is composed of tubes that are distributed in a polygonal pattern at a predetermined distance from the vessel wall. Such configuration indicated an up-flow of the fluid in the middle and a recirculation down-flow in the annular volume between the pulse tubes and the vessel wall. Hence the direction of the impeller pitch was reversed for an upward flow in the middle of the vessel to resemble the pulsed jet mixing flow pattern. 
Some assumptions in opting for a well-mixed vessel for testing were taken and are specified as follows:

- A well mixed condition provides bounding conditions of gas release for comparison of simulants.

- $\quad$ Gas injection with controlled bubble size provides a measure of the steady state hold - up and release rates. Computations had indicated that gas bubbles approximately $1.0 \mathrm{~mm}$ in diameter will freeze in place (or simulant "gels" instantly relative to bubble buoyancy forces) when mixing is stopped.

- A well stirred system provides a platform for rapid relative evaluation of simulant behavior. 


\subsection{TEST APPARATUS FOR GAS INJECTION EXPERIMENTS}

A diagram of the experimental test set - up used for the argon injection tests is given in the following figure.

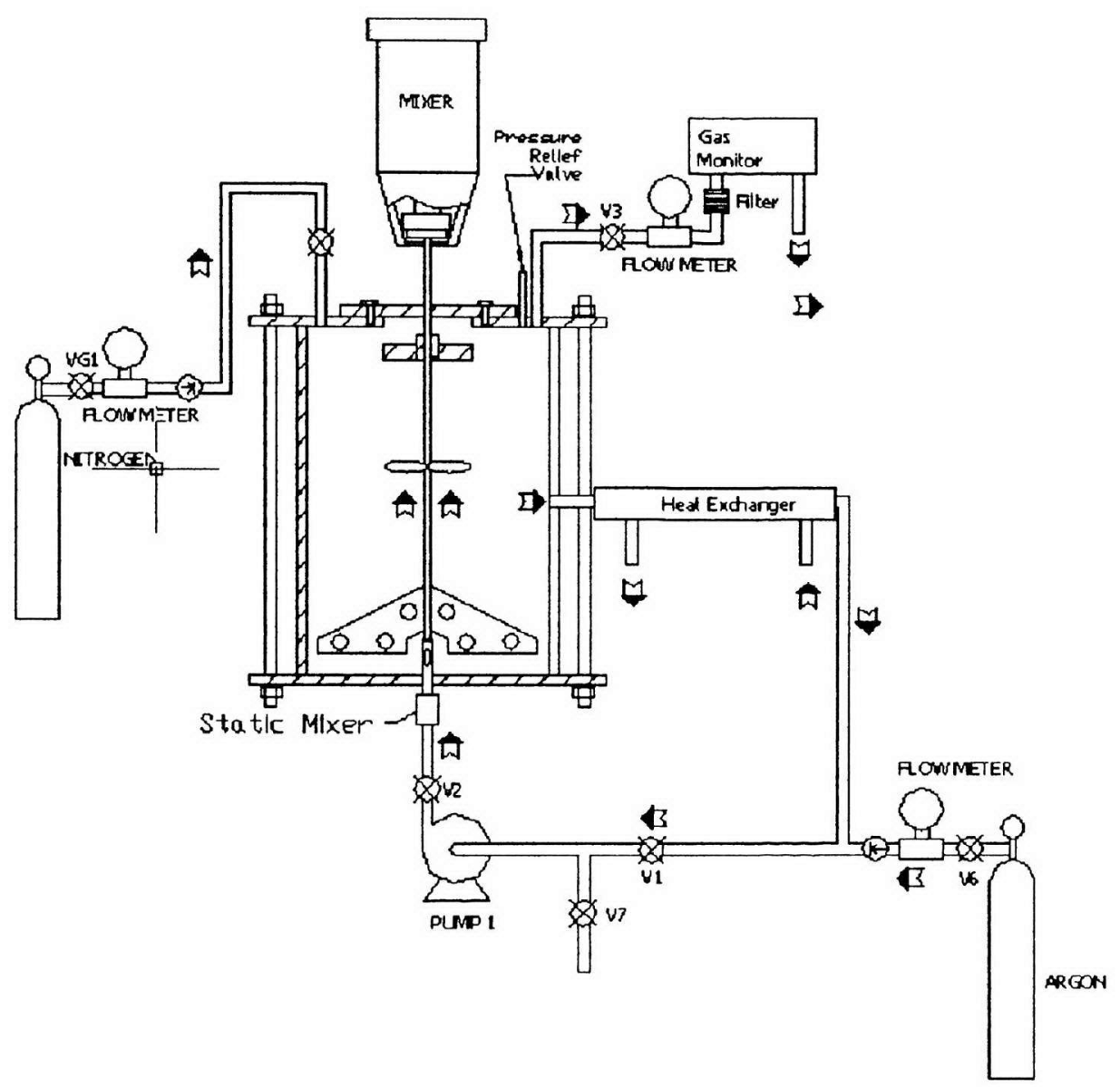

Figure 5 Experimental Setup for Study of Gas Retention and Release Behavior of Non Newtonian Simulants

The test set up was composed of two parallel primary test vessels with separate mixers that shared the feed gas (tracer and purge gas) system and the effluent gas 
analyzer. Furthermore, the vessels shared the mixer speed controller and monitoring tachometer. Consequently, at any given time, only a single vessel was operational.

\subsubsection{TEST VESSEL}

This unit was constructed using a section of acrylic pipe with 11.5 -inch inside diameter and 15-inches high. The vessel capacity was sized to allow 5 gallon charge of simulant with approximately $30 \%$ headspace above the simulant. Penetrations in the sidewall were provided for recirculation flow intake and for thermocouple to measure the simulant temperature. The top and the bottom of the vessel were made of 0.5 inch thick acrylic blind flanges. While the bottom flange was centered to the vessel to form an integral structure with the sidewalls, the top was held by O-ring compression seal. The compression was provided by 4 tie - rods connecting to the top and bottom flanges. A graphite - packed seal (similar to pump seals) was installed in the top flange for entry of the mixer shaft into the vessel. The body of the seal was custom fabricated from Teflon stock.

\subsubsection{MIXER}

The mixer consisted of a $0.5 \mathrm{HP}$ variable - speed DC motor electronically controlled that powered 4.5 inch diameter impellers specially designed for mixing high viscosity fluids. In addition to the two impellers, a "rake" or sweep - impeller was added below to the lower impeller to improve mixing in the volume below the lower impeller. In conceiving the design, it was assumed that enough mixing of the volume below the lower impeller could be obtained through transfer of momentum of the recirculation flow, 
facilitated by the radial deflector located at the bottom of the vessel. However, during the shake down tests with Laponite, it became obvious that the mixing cavern did not fully extend to the portion of the vessel below the lower impeller. Consequently, especially design rake was added to the mixer shaft below the lower impeller to allow total volumetric mixing of the simulant.

This sweep impeller shown in the following figure had a diameter of 9.75 inches with overall vertical dimensions of 3 inches. The impellers were located on a single $1 / 2$ inch shaft supported at the top by the packed seal and at the bottom by a Teflon bushing located at the top of the radial flow deflector. The shaft was driven through a flexible coupling to allow for ease of alignment of the motor drive and the shaft. The pitch of the impellers was selected for upflow displacement of fluid during mixing, and the impellers were located on the shaft to avoid gas entrapment in the vortex under well-mixed conditions. The mixer shaft speed (RPM) was monitored continuously with laser tachometer and controlled manually. 


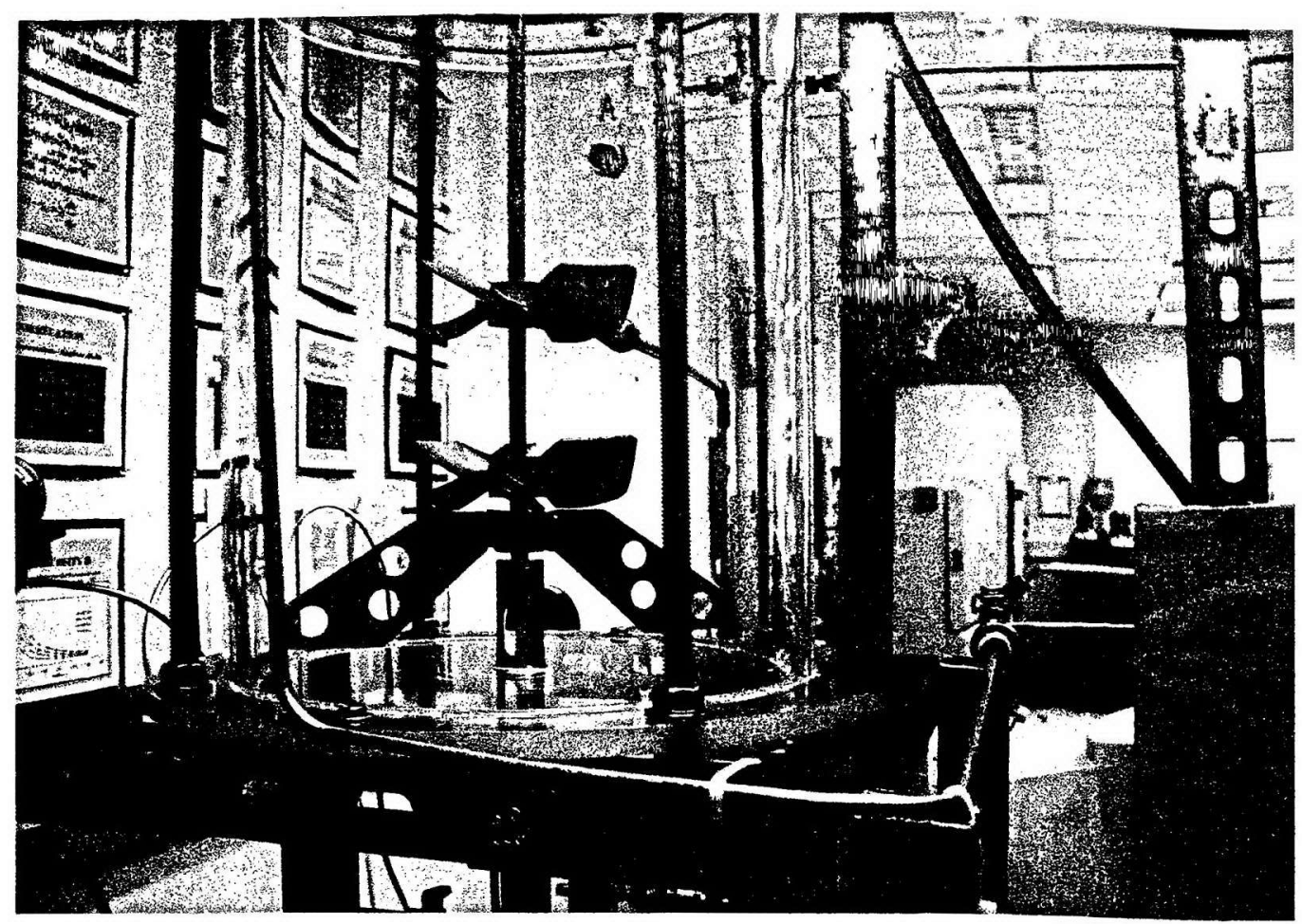

Figure 6 Test Apparatus Showing Test Vessel with Mixer Rake Modification

\subsubsection{PUMPS}

A variable speed positive displacement gear pump capable of 12 GPM throughput was used for the simulant recirculation. The injected gas was dispersed in an in-line static mixer downstream of gas injection and before the simulant vessel. The large bubbles are sheared in the static mixer into smaller bubbles. The control of the maximum bubble size was achieved by controlling the recirculation flow rate through the static mixer. The minimum simulant recirculation rate required to obtain the acceptable bubble size $(<1 \mathrm{~mm}$ diameter) was judged using $30 \mathrm{~Pa}$ laponite simulant. The bubble size was determined by the size of the bubbles near the wall vessel, measured by visual observation and a centimeter - scale attached to the vessel wall. This can be shown on the following figure. 


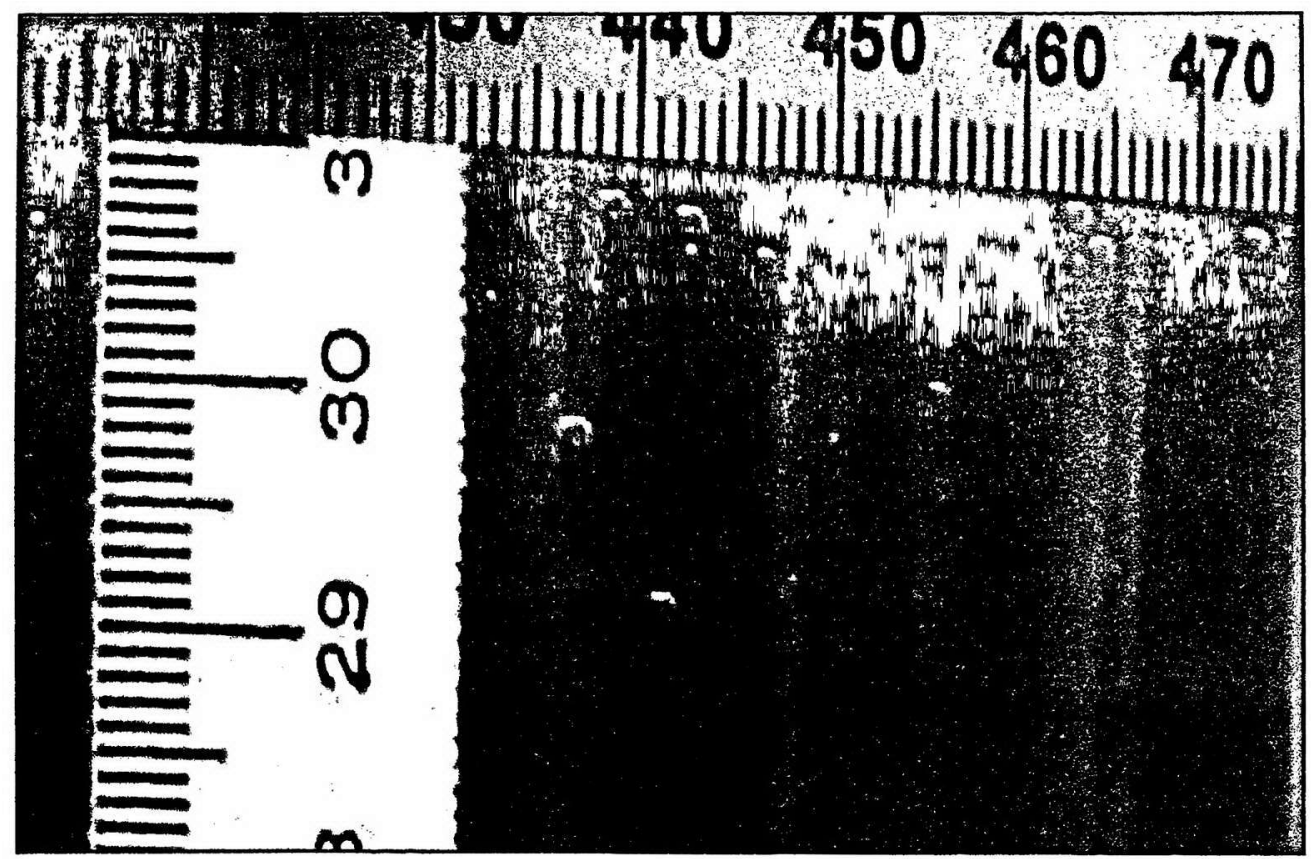

Figure 7 Gas bubbles dispersion in Laponite simulant

Higher recirculation rates resulted in smaller bubble size because of greater fluid - shear in the static mixer. The injection rate of argon was monitored and recorded with and electronic flow controller connected to the data acquisition system. The dispersed argon was introduced into the vessel below the impellers through a radial flow deflector.

\subsubsection{HEADSPACE}

The headspace in the vessel above the simulant was purged with nitrogen at a flow rate with approximately 1.5 liters/min. both the inlet and outlet for the purge were through the top flange located diametrically across in a hole - circle diameter of about 9 inches. Nitrogen was introduced from a supply cylinder through an electronic flow controller connected to the data acquisition system. The purge rate was monitored and 
recorded throughout each test. A similar arrangement was set up to measure and record the amount of gas exiting the vessel.

\subsection{TEST REQUIREMENTS FOR GAS RETENTION AND RELEASE EXPERIMENTS}

The following requisitions need to be accomplished when setting up the test (WTP predetermined set conditions): approximately 5 gallon range on test vessel, suitable mechanical agitator system to be defined and agreed upon with WTP, ability to inject gas near the axial flow impellor blades so as to 'beat' the gas bubbles into smaller bubbles and disperse them uniformly into the simulant and a millimeter wave level detection probe/system or other similar device, would need to be able to measure $1 / 2 \%$ level change in the vessel.

\subsection{TEST PROCEDURES FOR GAS RETENTION AND RELEASE EXPERIMENTS}

The procedure for these tests can be broken down into following operations: 1) System assembly, 2) Gas leak test, 3) Vessel filling, 4) Simulant conditioning, 5) Gas injection for steady state, 6) Gas injection for hold-up, 7) Gas release from steady state, 8) Gas release during simulant gelling, 9) Gas release from gelled state and 10) Equipment clean-out 


\subsubsection{SYSTEM ASSEMBLY}

The schematic diagram of the system assembly is shown in Figure 4. The procedure described here is limited to the simulant vessel and the associated hardware. These include: recirculation pump, static mixer, simulant impeller mixer, simulant level probe assembly.

- Ensure that all parts are clean and free of any oily residues or film.

- Clean the simulant level probe with alcohol or degreasing solvent to ensure good contact.

- Ensure positioning and orientation of the impellers. The bottom impeller should be located about $25 \%$ of the expected liquid level height from the vessel bottom, and the top impeller about $75 \%$ of the height from the bottom. Both impellers should be oriented to move flow upwards.

- Ensure the O-ring groove for the top flange seal is clean. Check the ring seating at the top flange before tightening the flange bolts. Do not over tighten these bolts. Follow torque ratings for the bolts if available.

- After assembly, turn on the impeller mixer gradually to make sure of no loose parts or vibrations. Set the mixer motor direction. 


\subsubsection{VESSEL FILLING}

This section provides the procedure used for filling the vessel with the simulant and measuring the initial level of the simulant in the vessel:

- Close all the valves leading to and out of the simulant vessel.

- Remove the top flange with its associated components (mixer assembly, simulant level probe etc.) and fill the vessel with approximately 5.5 gallons plus estimated inventory of simulant in lines, pump and static mixer recirculation loop. Exercise care not to entrap any air into the simulant during this operation.

- Clean the O-ring seating groove and re-assemble the top flange assembly to the vessel.

- Carry out gas leak check.

- Record the simulant level using both the simulant level probe and the measurement scale on the side of the vessel.

- Start the mixer and increase the speed very gradually to avoid cavitation or cavern formation. Increase the mixer speed until the predetermined mixing speed is reached. Verify visually that there is visible movement of liquid over the entire top surface and the wall area. Continue mixing the simulant for at least 1 hour at this speed to fully shear the simulant inventory.

- Stop mixer and verify there is no air entrapment and let the surface level out. Record simulant level in the vessel using both the level probe and the scale on the vessel.

- With the impeller mixer turning slowly to facilitate gas escape, start the recirculation pump very slowly and purge the air in the pump, static mixer and the lines. 
- After line purge with simulant, increase the recirculation flow rate to the rate determined after performing the static mixer calibration to generate the desired gas bubble size. Continue recirculation for at least 30 minutes to purge out any air entrapped in the lines.

- Remove any air entrapped in the simulant by bumping the mixer, if needed. Resume mixing the simulant in the vessel for 30 minutes. The process of "bumping" the mixer involves slowing down the mixer speed followed by a short period surge of mixing at a significantly higher speed.

- Once nearly all entrapped air has been released, shut down the mixer and the pump. Measure and record the simulant level in the vessel.

\subsubsection{GAS LEAK TEST}

- Isolate the simulant vessel from the remainder of the system by closing all the valves leading to and out of the vessel.

- Connect a manometer to the effluent gas line, with the one end open to the atmosphere.

- Connect the purge nitrogen gas source to the vessel and very slowly pressurize the gas to about 1.0 psig ( 27 inches of water or $5 \mathrm{cms}$. of mercury). Close-off the nitrogen purge line valve and allow the pressurized vessel to sit.

- Check all seals and connectors for any gross leak with commercially available leak detector solution (such as "Snoop") or a mixture of surfactant and water. 
- Record the manometer pressure drop reading every 15 minutes. If the pressure drop decreases by less than $10 \%$ of its initial value in $4 \mathrm{hrs}$, the system may be considered gas leak tight and ready for the gas retention and release test.

\subsubsection{SIMULANT CONDITIONING}

Simulant conditioning is essential before gas injection into the simulant. The following procedure is to be followed for conditioning the simulant:

- Record the simulant level using both the simulant level probe and the measurement scale on the side of the vessel.

- Start the mixer and increase the speed very gradually without cavitations or cavern formation. Increase the mixer speed until the predetermined mixing speed is reached. Verify visually that there is visible movement of liquid over the entire top surface and the wall area of the vessel.

- Start the recirculation pump and increase the recirculation flow rate to the rate determined in after performing the static mixer calibration to generate the desired gas bubble size.

- Continue mixing the simulant with recirculation for at least 1 hour.

- Stop mixer and pump briefly and long enough to verify there is no air entrapment and to let the surface level out. Record simulant level in the vessel using both the level probe and the scale on the side of the vessel.

- Resume simulant mixing and pump recirculation. The system is ready for gas injection. 


\subsubsection{STEADY STATE HOLD-UP AND RELEASE TEST}

\subsubsection{GAS INJECTION FOR STEADY STATE HOLD -UP}

The steady state gas hold-up in the simulant is reached when the amount of argon in the effluent stream equals that injected (this being measured over a length of time). This can be established by verifying the concentration of argon in the effluent gas equals the estimated concentration of argon based on the argon injection rate and the nitrogen purge gas rate over at least a 30 minute period.

- Start the mixer and the recirculation pump and precondition the simulant.

- Start and set the purge gas (nitrogen) rate to $1000 \mathrm{ml} / \mathrm{min}$. Continue the purge until the nitrogen concentration in the effluent gas is greater than $98 \%$.

- Start argon injection at $40 \mathrm{ml} / \mathrm{min}$. Observe the bubble size. If the bubbles seem larger than $1 \mathrm{~mm}$ in diameter, increase the recirculation rate gradually until the maximum bubble size is smaller than $1 \mathrm{~mm}$ in diameter.

- Maintain constant mixing, recirculation and gas injection rates. Monitor the argon concentration in the effluent gas stream every 5 minutes.

- Upon reaching the steady state, shut off gas injection, mixing and simulant recirculation. Measure and record the simulant level and estimate the steady state gas hold-up in the simulant from the level measurements before and after gas injection.

- Maintain the nitrogen purge gas rate, and monitor the effluent gas flow rate and the argon concentration in the effluent gas. 


\subsubsection{GAS RELEASE FROM STEADY STATE HOLD-UP}

The objective of this task is to monitor and measure the release of gas from the simulant, once the gas hold-up has reached the steady state.

- Inject gas into the simulant at $40.0 \mathrm{ml} / \mathrm{min}$ until steady state is reached and with the mixer shut-off, record the simulant level

- Start the mixer at the predetermined rate. Do not resume gas injection and simulant recirculation.

- Monitor and measure the argon and nitrogen concentrations in the effluent stream from the time the mixer is turned on.

- Also, monitor and measure the nitrogen purge gas rate and the effluent gas rate.

- Stop the test when the release rate has reached an asymptotic value and remained there for a 2 hour period.

\subsubsection{PREDETERMINED GAS HOLD-UP AND RELEASE TEST}

\subsubsection{GAS INJECTION FOR PREDETERMINED HOLD-UP}

The gas hold-up in the simulant is determined by change in the volume of the simulant in the vessel. It is understood that the accuracy of this measurement is limited by the ability to measure the simulant level in the tank. The procedure in this task does not require steady state conditions to be achieved.

- Record the simulant level in the vessel.

- Start the mixer and the recirculation pump and precondition the simulant. 
- Start and set the purge gas (nitrogen) rate to $1500 \mathrm{ml} / \mathrm{min}$. Continue the purge until the nitrogen concentration in the effluent gas is greater than 98 percent.

- Start argon injection at $150 \mathrm{ml} / \mathrm{min}$. Observe the bubble size. If the bubbles seem larger than $1 \mathrm{~mm}$ in diameter, increase the recirculation rate gradually until the maximum bubble size is smaller than $1 \mathrm{~mm}$ in diameter.

- Monitor and record the purge gas rate, argon injection rate, the effluent gas rate and the effluent argon concentration throughout the test.

- Maintain constant mixing, recirculation and gas injection rates. Monitor the simulant level in the vessel every 5 minutes while mixing. It is acknowledged that the accuracy of this interim level measurement is compromised by gas injection, mixing and simulant recirculation. Therefore, to obtain the final hold-up level, it is required to shut-off gas injection, mixing and simulant recirculation.

- Stop gas injection, mixing and simulant recirculation when the simulant level is believed to slightly exceed the desired hold-up.

- Maintain the nitrogen purge gas rate and monitor the effluent gas rate and composition for argon.

- Measure and record the final hold-up level. 


\subsubsection{GAS RELEASE FROM PREDETERMINED HOLD-UP}

The objective of this task is to monitor and measure the release of gas from the simulant, once the gas hold-up has reached the predetermined level.

- Complete gas injection into the simulant at predetermined rate until the desired holdup is reached.

- Start the mixer at the predetermined rate. Do not resume gas injection and simulant recirculation.

- Continue to monitor and record the argon and nitrogen concentrations in the effluent stream from the time the mixer is turned on.

- Monitor and record the nitrogen purge gas rate and the effluent gas rate.

- Stop the test when the release rate has reached an asymptotic value and remained there for a 2 hour period.

- Measure and record the simulant level, and estimate the residual gas hold up.

\subsubsection{SIMULANT GELLING AND GAS RELEASE}

\subsubsection{GAS RELEASE DURING SIMULANT GELLING}

Once the gas injection, mixing and simulant recirculation have ceased, the simulant gelling process was started. The gelling occurs gradually for each simulant with an associated time constant to reach its full yield stress. The gas release from the simulant continues until the simulant has gelled sufficiently to entrap the gas from migrating to the surface. Therefore: 
- When the hold-up has reached the desired level, shut-off gas injection, mixing and simulant recirculation. Maintain the purge gas flow.

- Continue to monitor and record the purge gas and effluent gas flow rates, and argon and nitrogen concentrations in the effluent gas until the argon concentration drops off to a low asymptotic value over a 2 hour period.

- Measure and record the simulant level and estimate the gas hold up.

\subsubsection{GAS RELEASE FROM GELLED SIMULANT}

This procedure follows gas release monitoring from the gelling simulant.

- Shut-off the nitrogen purge gas and close-off all the valves leading in and out of the vessel to isolate the simulant. Let the simulant gel overnight.

- Open the valves for the purge gas line and the effluent gas lines. Set the purge gas rate at $1500 \mathrm{ml} / \mathrm{min}$. Measure and record the argon accumulated overnight in the overhead space in the vessel. Continue this until the argon concentration reaches the background level.

- Start the mixer and gradually increase the mixer speed to the predetermined value from Section 2.

- Continue to monitor and record the purge gas and effluent gas flow rates, and argon and nitrogen concentrations in the effluent gas until the argon concentration drops off to a low asymptotic value over a 2 hour period.

- Measure and record the simulant level and estimate the gas hold up. 


\subsubsection{RHEOLOGY SAMPLES}

The test plan requires that for each test completed, a 1 liter sample of the simulant before the test, and a 1 liter sample of the simulant collected from the vessel after the test be collected in polyethylene or polypropylene bottles with leak tight screw caps.

Each sample should clearly indicate the following information on its label: Sample Number, Simulant Identification, Date and Time of Collection.

\subsubsection{STEADY STATE TESTS}

\subsubsection{RELEASE FROM STEADY STATE RETENTION}

As specified earlier, the steady state tests consisted of two parts: steady state retention and release, and release from the simulant gelled from the steady state condition.

For the first stage of the experiments argon at predetermined injection rate was introduced into simulant as small bubbles mostly less than $1.0 \mathrm{~mm}$ in diameter. The injection was maintained at the controlled injection rate until the system reached steady state. The effluent gas from the system was analyzed by continuous gas phase mass spectrometer for argon and nitrogen. The periodic concentration values at discrete times were recorded in the data acquisition system.

The system was maintained at steady state for approximately 30 minutes before curbing argon injection to measure the release rate. Tests were carried out with both the clay and QARD simulant at two levels of argon injection: $40 \mathrm{ml} / \mathrm{min}$ and $160 \mathrm{ml} / \mathrm{min}$. 
The maximum hydrogen generation rate in WTP waste is estimated to be 0.001 moles $\mathrm{H}_{2} / \mathrm{kg}$.waste/day (Sherwood et al. 2004). This value corresponds to 22.4 $\mathrm{ml} / \mathrm{kg}$.waste/day at 20 degree Celsius and 14.7 psia pressure. For a waste set of 5.5 gals and a waste specific gravity of 1.23 , the gas concentration rate is estimated as $0.4 \mathrm{ml} / \mathrm{min}$. the lower level of argon injection ( $40 \mathrm{ml} / \mathrm{min}$ ) was selected to be 100 times that of the hydrogen generation rate. It was assumed that this most likely provide a lower bound for steady state hydrogen retention for the current tests. The higher level of injection at 160 $\mathrm{ml} / \mathrm{min}$ was used to test for the maximum gas retention capacity of the simulant.

Once having operated for approximately 30 minutes at steady state, the release phase of the test was initiated by stopping the argon injection and simulant recirculation. During this phase the mixing was continued along with the purged of the headspace and effluent gas monitoring until the argon concentration reached the lower measurement limit of the gas analyzer. The release stage lasted approximately between 60 to 180 minutes.

\subsubsection{RELEASE FROM GELLED STEADY - STATE HOLD - UP}

Following having reached the steady state retention level, the simulant was allowed to gel by shutting off both argon injection as well as the mixing. Because of the small bubble size selected, the bubbles freeze within the simulant within a few seconds after the mixing is stopped. In other words, for the bubble size range used for testing, the simulants gels immediately after the mixing is stopped. 
Even if the mixer and injection were shut down, flow of purge gas and analysis of the effluent was composition were performed until the argon concentration reached the lower measurement limit. This permitted done to account for argon in the headspace at the time of injection shutdown and for that released from the simulant during the initial gelling period. This period lasted no more than 30 minutes. The vessel was afterwards isolated by closing off all inlet and outlet valves, and the simulant continued to set overnight for a total gelling period of eighteen hours. The mixer shaft seal was checked for gas leak, and tightened before isolating the vessel.

The gel release was initiated by the commencement of mixing of the simulant. However, prior to this argon released over the 18 hour period and collected in the headspace was purged and analyzed. Once it is certain that the headspace is clear of argon, mixing is initiated to measure the gel release rate. The release of argon was monitored until the argon concentration reached the minimum measurement level in the effluent gas.

The gel release tests, therefore, have four separate periods for argon monitoring: attainment of steady - state retention, release during initial gelling period, release during overnight 18 hour gelling period, and gel release upon mixing the gelled simulant.

\subsection{SIMULANT RHEOLOGY}

Besides laponite, a $27 \%$ Clay mixture suspension and a precipitated hydroxide simulant (QARD) were used in the test for steady state retention and release. A brief of the simulant rheology for the Bingham plastic model fit is given below. The rheology 
determinations of the simulants before and after the end of the test program were carried out by Savannah River National Laboratory (SRNL) (P. Sundar 2004).

Table 1 Rheology of Simulants Employed in Steady State Retention and Release Tests

\begin{tabular}{|l|l|l|}
\hline Simulant & Bingham Plastic & Bingham Plastic \\
\hline Laponite before testing & 7.64 & Viscosity, cP \\
\hline Laponite after testing & 7.63 & 8.88 \\
\hline 27\% Clay before testing & 16.24 & 9.31 \\
\hline 27\% Clay after testing & 12.04 & 24.48 \\
\hline QARD before testing & 3.39 & 27.70 \\
\hline QARD after testing & 16.38 & 15.62 \\
\hline
\end{tabular}

It can be clearly noted in figures 8 and 9 how the simulant behaviour of the simulants before and after testing resembles the viscoplastic fluid behavior of a Bingham fluid where the fluid is characterized by a constant plastic viscosity and a yield stress. 


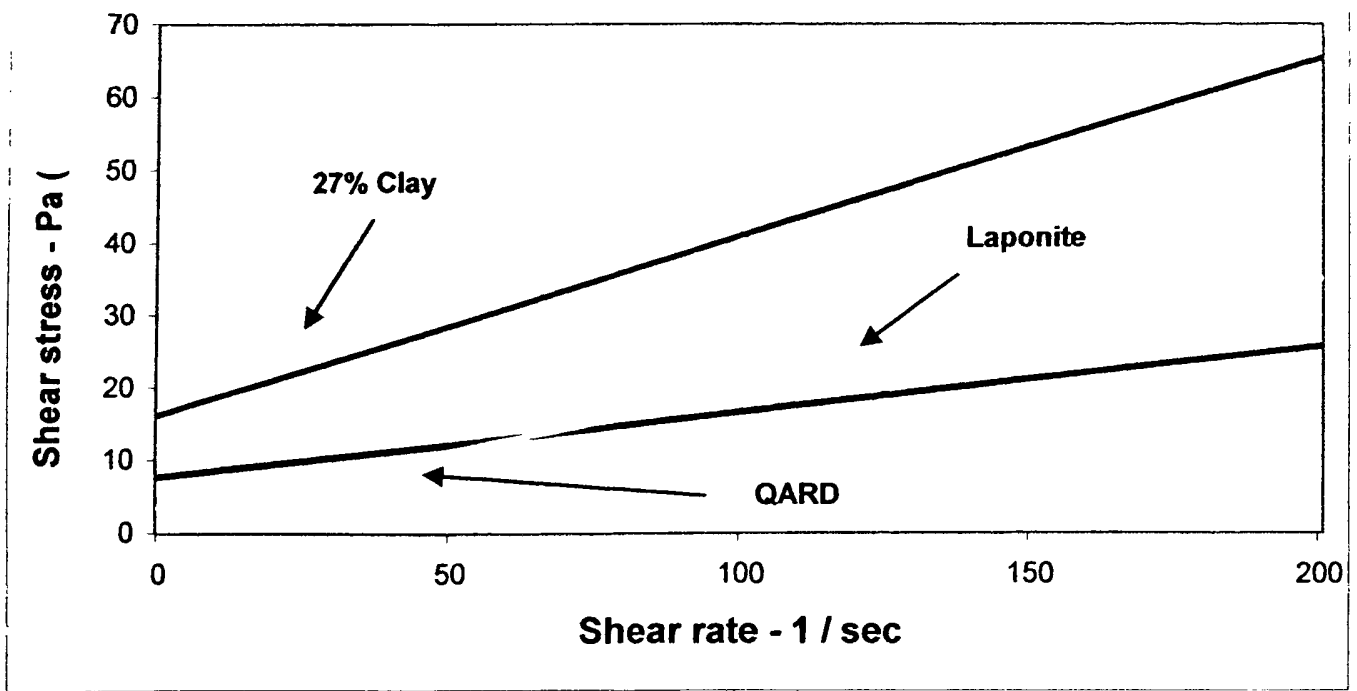

Figure 8 Shear stress - shear rate behaviour of simulants before testing

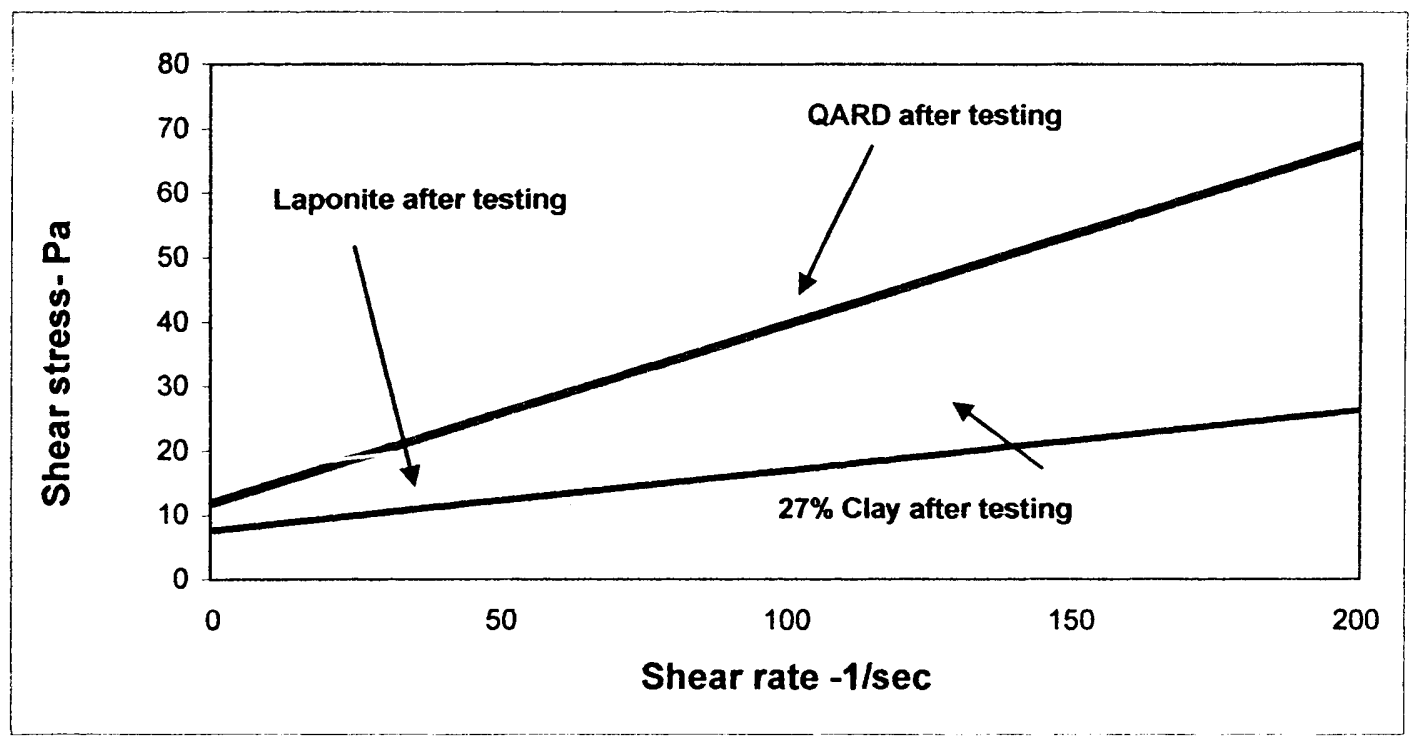

Figure 9 Shear stress - shear rate behaviour of simulants after testing 
For the duration of the steady state tests, the variables measured were the following: argon and nitrogen flow rate into the vessel, argon and nitrogen concentration of the effluent gas. Also, the temperature of the simulant was recorded routinely as well as the initial simulant and headspace volumes in the vessel. All the collected data was used to estimate the volume of the gas retained in the simulant during steady state period and the release rate from the simulant during the release phase from steady state and gelled state respectively.

\subsection{EXPERIMENTAL OBSERVATIONS}

\subsubsection{LAPONITE STEADY STATE INJECTION TEST}

The laponite simulant in the system shakedown tests was a clear blue suspension. This clearness of the simulant allowed visual observation of the interior of the simulant volume and was the principal reason for its use for shakedown testing.

The laponite test sought to: (i) calibrate the simulant recirculation rates required to provide less than $1.00 \mathrm{~mm}$ diameter bubbles at the planned test injection rates, (ii) verify the mixing pattern and determine the mixer RPM range for the tests, and (iii) verify operability of the conductivity probe. Due to these tests performed with laponite some changes to the system were made and are specified as follows: removal of the baffles in the vessel provided to improve mixing, addition of specially design rake, addition of a chiller to maintain the simulant temperature since an increased of about $10 \mathrm{deg} \mathrm{C}$ was 
observed. Due to the temperature increment an in line coolant jacket was added to the simulant recirculation line to maintain constant simulant temperature.

The following observations were made with the laponite: the surface vortex was very stable for a given mixer speed, a bubble size $<1.00 \mathrm{~mm}$ could be achieved with the system as configured even at the high argon injection rate of $150 \mathrm{ml} / \mathrm{min}$. The simulant level change during the test was not sufficient to measure gas retention or the gas retentions were too low to measure through level change. The spatial density of bubbles in the simulant observed upon ceasing injection and mixing was low and seemed consistent with little measurable level change and the retention may have been lower at the higher injection rate because of loss of control over the bubble size and due to increased bubble coalescence in the simulant. Increased injection seemed to increase gas coalescence in the vicinity of the mixer shaft in the vessel. The coalescence gas appeared to escape continuously in an upflow stream around the mixer shaft. Therefore, an attempt to test the retention capacity of the simulant by increasing the injection rate was not successful. Since the direct measurement of holdup gas could not be established. The holdup has been determined by the theoretical approach explain earlier.

When the steady state test were performed with Laponite, this tests required the least amount of adjustments to the mixing speed to maintain the surface vortex profile during the test, once it has been set at the start of the test. In figure 7 we can observe the fluctuations in the injection period that reflect the rheology changes during injection. 


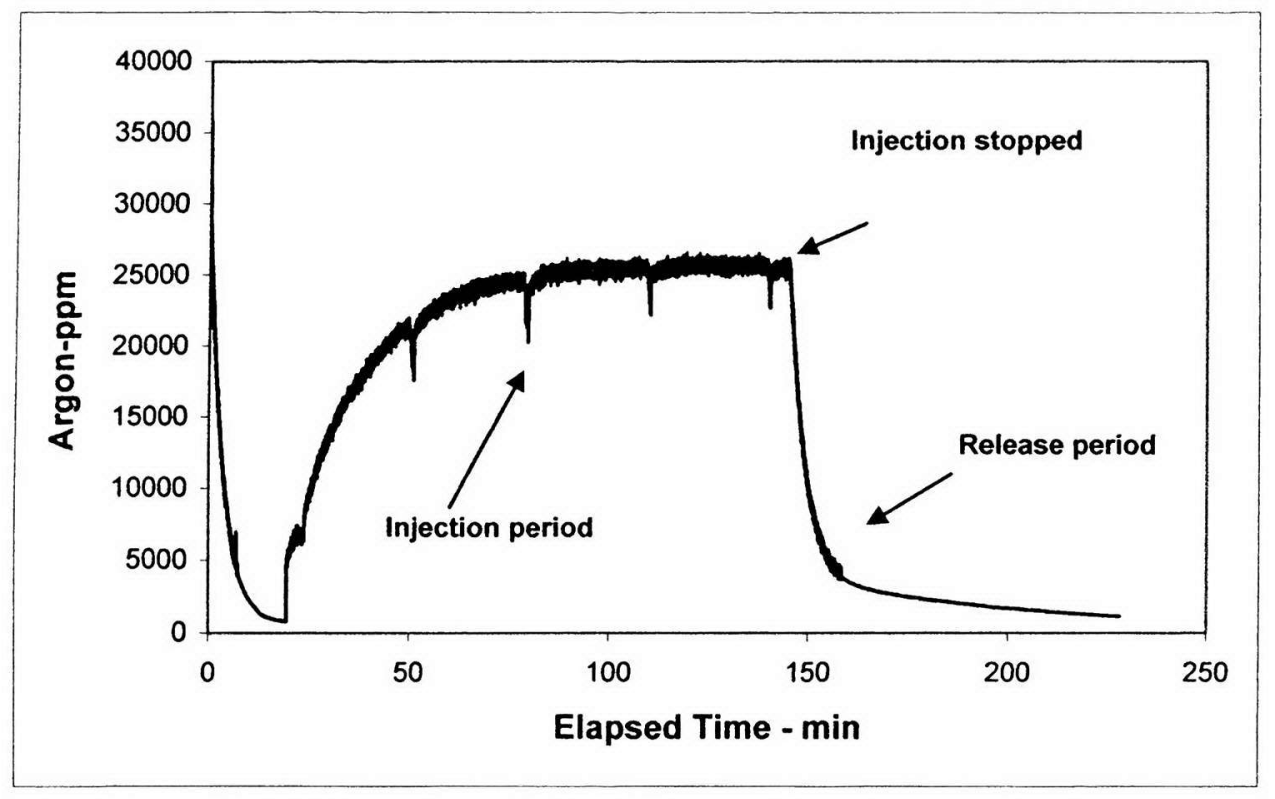

Figure 10. Argon Concentration During Release from Steady State with Laponite Simulant

\subsubsection{DILUTED CLAY STEADY STATE INJECTION TEST}

When performing this test it was noted that a greater amount of adjustments to the mixing speed were required to maintain the surface vortex profile required. Clay simulant seemed to accumulate argon and release it in intermittent surge of gas release. This behavior was noted as a gradual rise in the center of the vortex followed by a collapse.

The simulant appeared to become more thick and viscous as the injected gas was being incorporated. This was observed by the need of continuously increasing the mixer speed to maintain both the surface vortex profile as well as the fluid movement at the wall. The third significant observation was the premature wear and failure of the recirculation pumps due to the abrasive character of the simulants. The erosion of the 
pump during the injection period was sufficient to change pump characteristics drastically. This could have resulted in a larger bubble size. The observed intermittent surges of argon concentration in the effluent gas during injection was attributed to periodic adjustments to the mixer speed and premature erosion of the pump leading to larger bubble size the unstable argon retention during the injection period can be clearly noted in the figure below.

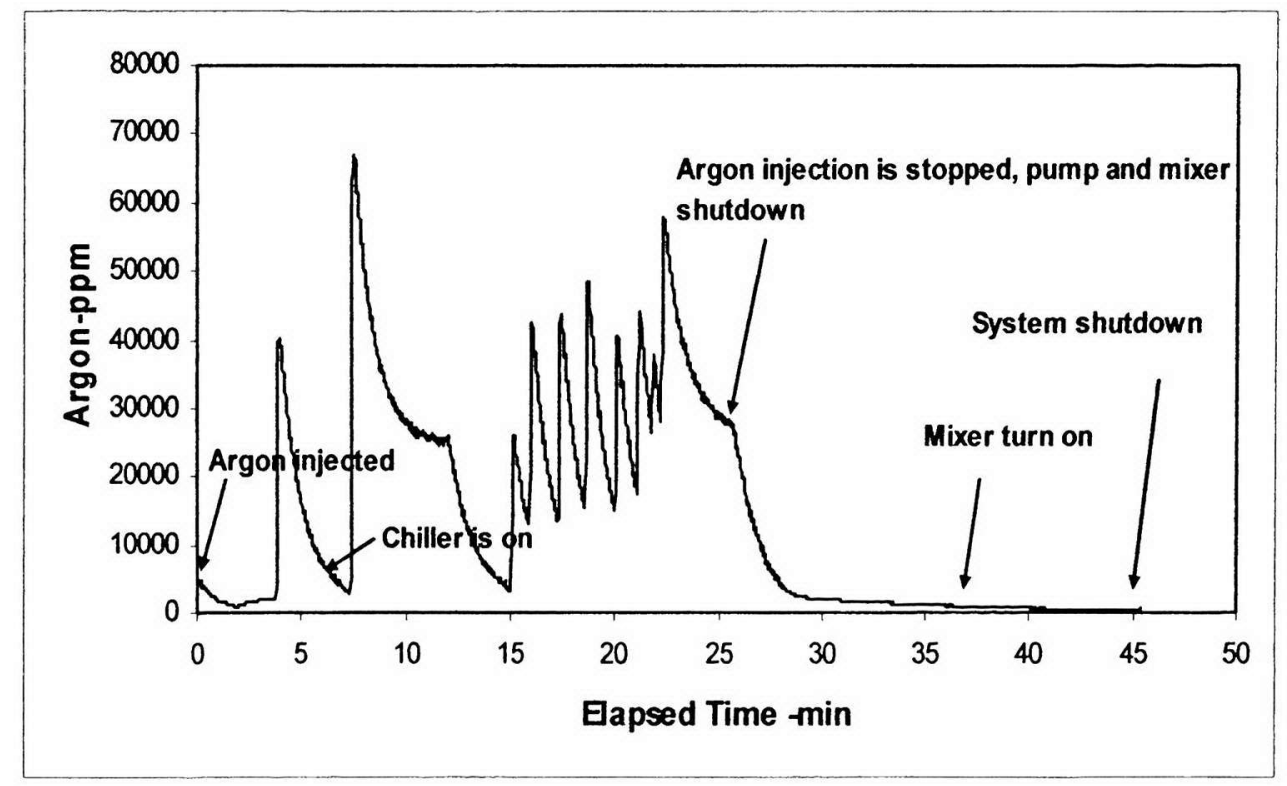

Figure 11 Argon Concentration during Release from Steady -State Retention with Clay Simulant

A mass balance was performed in the entire system using the analytical model explained above to determine the percentage of holdup gas in the simulant. Figure 9 shows the results obtained from the mass balance performed in the system. It can be noted from figure 9 that the percentage of holdup gas is approximately $5 \%$. 


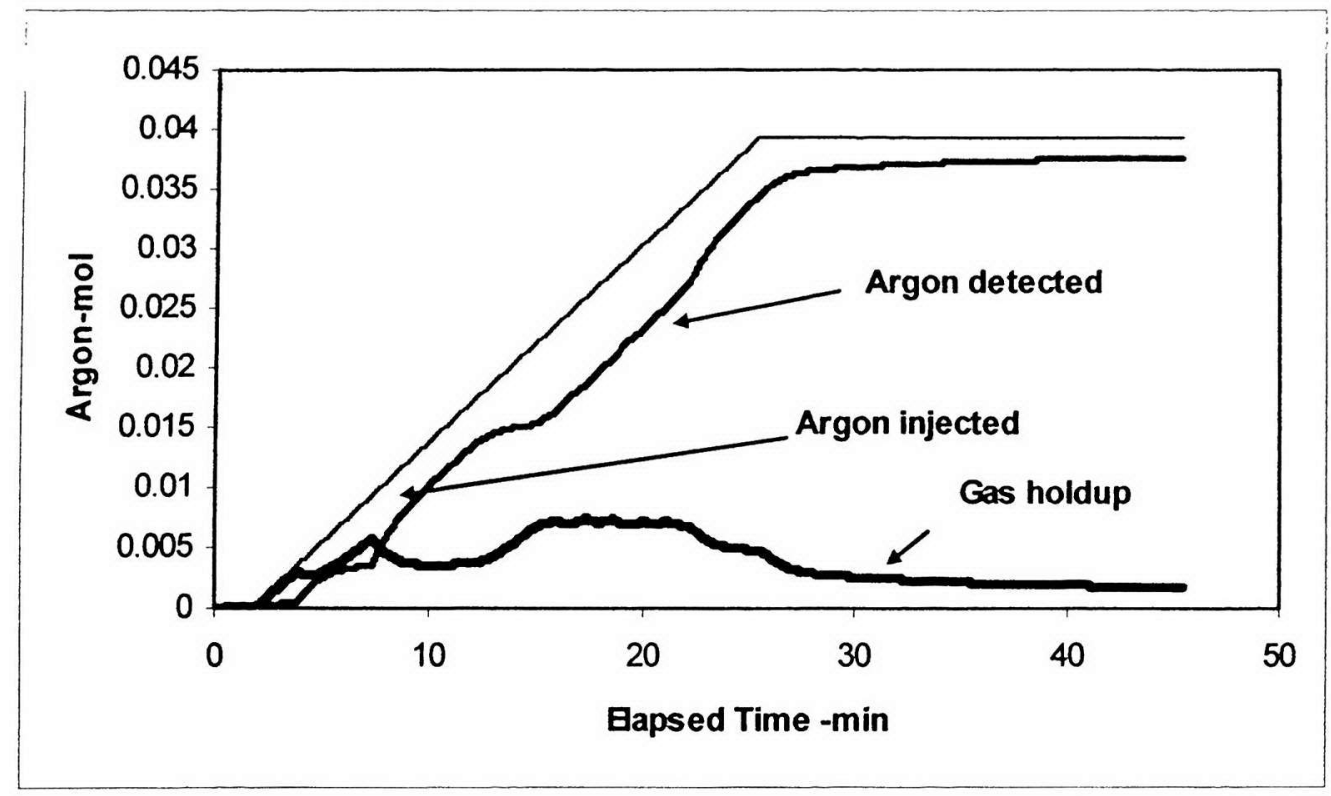

Figure 12 Gas Holdup Detected in the diluted Clay Simulant after Steady - State Injection Test

\subsubsection{CLAY $27 \%$ STEADY STATE RELEASE TEST}

When performing this experiment the same observations made in the experiment with the diluted clay were made. In the Figure 10 below the fluctuations in the injection period can be observed as well. The unstable retention in the simulant during the injection period can be clearly noted. 


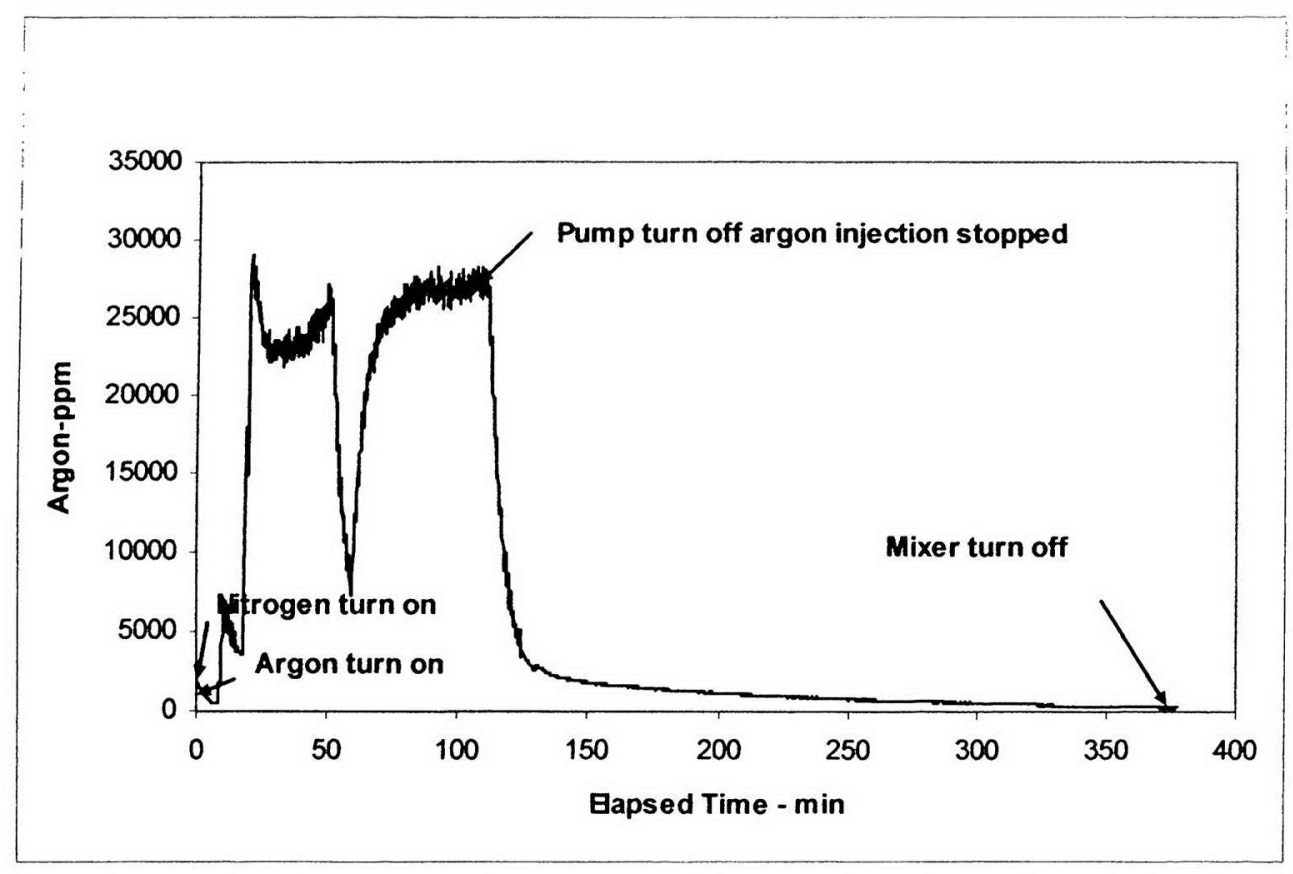

Figure 13 Argon Concentration during Release from Steady - State retention with $27 \%$ Clay Simulant

The rate of release has been determined from Figure 10 and has been shown in the following figure. Clearly three zones can de noted the first one relates to the smallest bubbles coming out of the simulant, the second zones relates to bigger bubbles coming out and the third one relates to the accumulated gases trapped in the simulant.

In the figure below it is shown the different zones encountered in the steady state release from $27 \%$ Clay. 


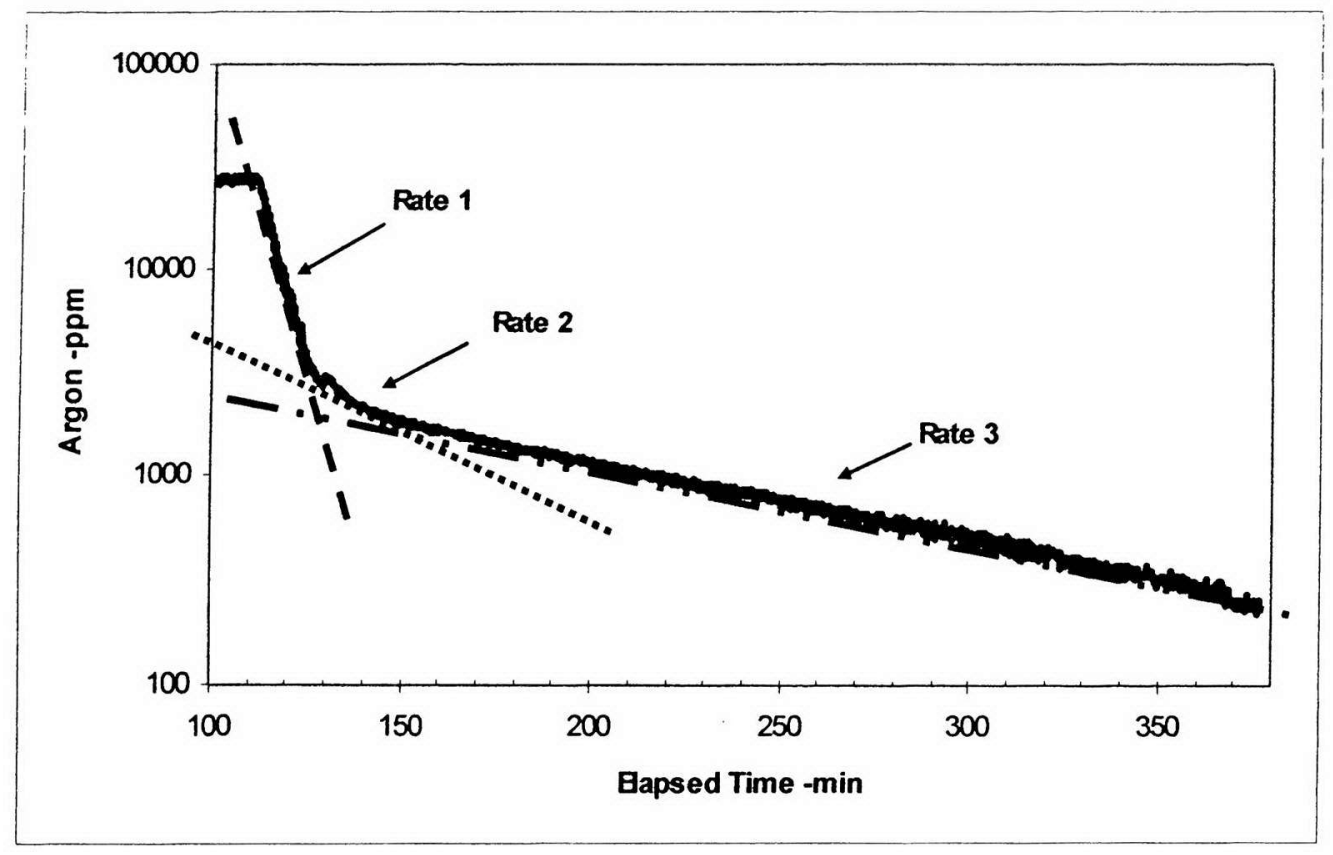

Figure 14. Rate of Release from 27\% Clay during Steady State Release 
The rate of holdup was determined from figure 11 and tabulated in the following table:

Table 2. Rate of Release from $27 \%$ Clay during Steady State Release

\begin{tabular}{|c|c|c|c|c|}
\hline $\begin{array}{c}\text { Time (X1) } \\
(\min )\end{array}$ & $\begin{array}{l}\text { Argon Detected } \\
\text { (Y1) (mol) }\end{array}$ & $\begin{array}{c}\text { Time (X2) } \\
(\min )\end{array}$ & $\begin{array}{l}\text { Argon Detected } \\
\text { (Y2) (mol) }\end{array}$ & $\begin{array}{c}\text { RATE OF } \\
\text { RELEASE (R) } \\
\text { R= (Y2-Y1)/(X2-X1) } \\
(\mathrm{mol} / \mathrm{min})\end{array}$ \\
\hline 119.3 & 8709.3 & 123.4 & 5222.06 & -850.5463 \\
\hline 139.6 & 2137.45 & 141.8 & 2044.66 & -42.17727 \\
\hline 204 & 1164.44 & 216.3 & 1022.54 & -11.53659 \\
\hline
\end{tabular}

\subsubsection{QARD 13.5 STEADY STATE INJECTION TEST}

In this test $40 \mathrm{ml}$ of argon was injected into Qard 13.5 until reaching steady state condition. For the duration of the injection period it was observed as an unstable period due to the changes in the rheology of the simulant. These changes can be noted in figure 12. 


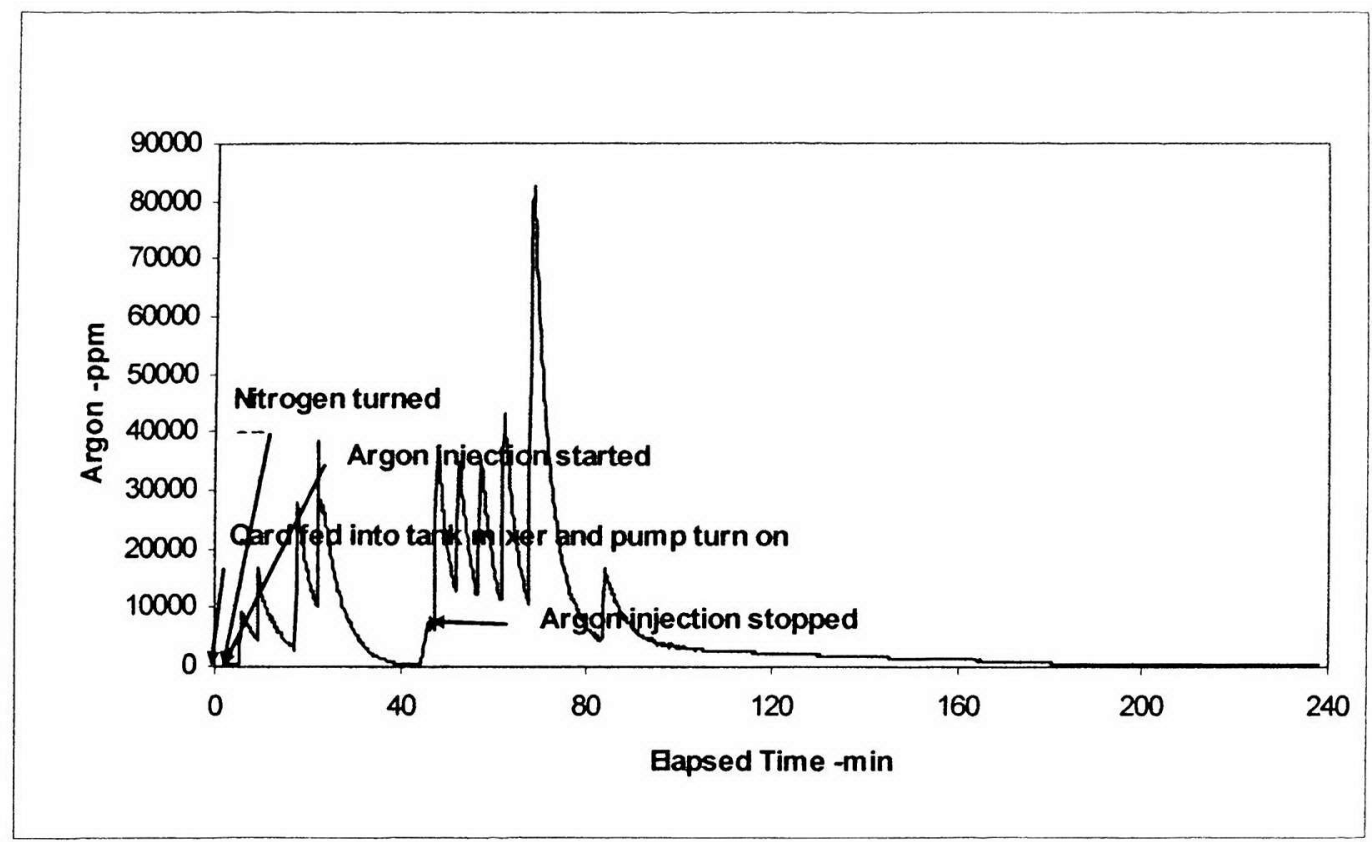

Figure 15 Argon Concentration during Injection into Qard 13.5 until reaching steady state condition.

A mass balance was calculated from the concentration of argon injected and detected through the gas analyzer. These calculations were performed in order to determine the percentage on argon holdup during injection in steady state condition. The percentage of gas holdup is about $5 \%$ during the injection period as can be noted in figure 13. 


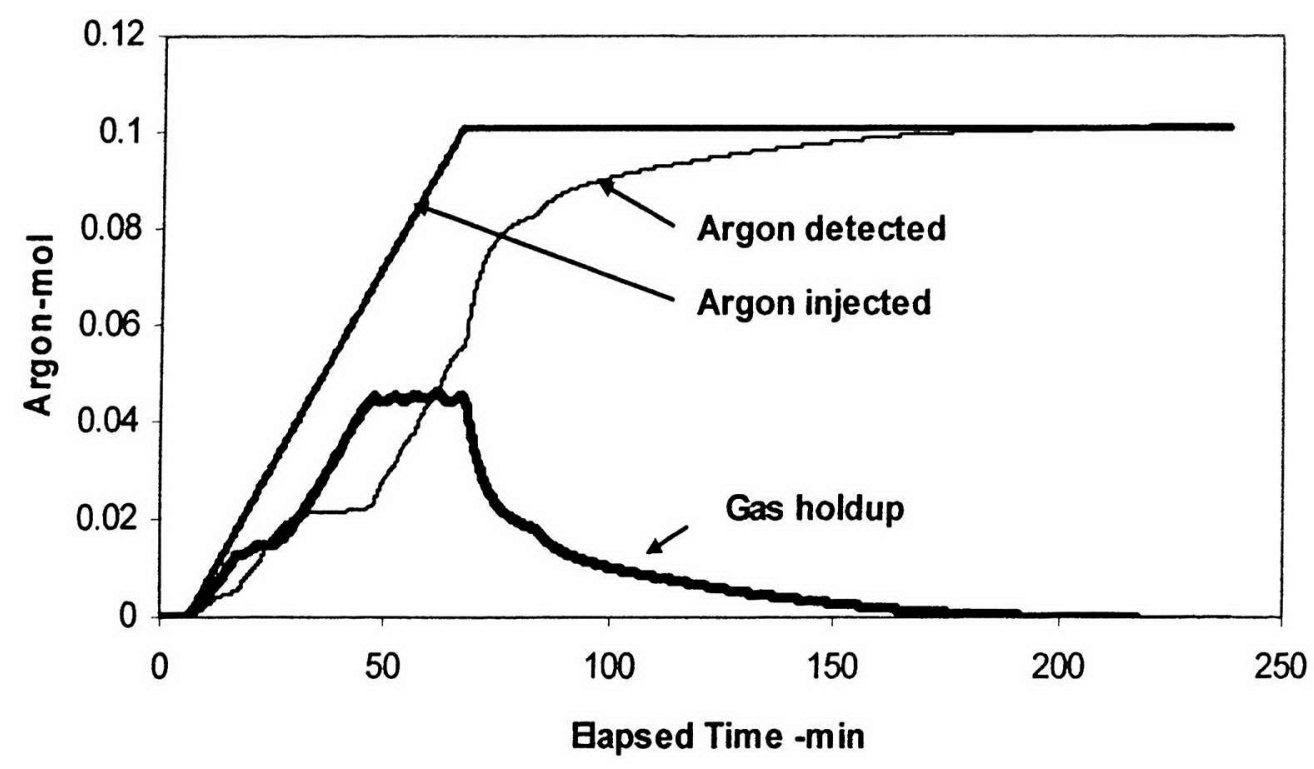

Figure 16 Gas Holdup Detected in the diluted QARD Simulant after Steady - State Injection Test

\subsubsection{QARD 13.5 STEADY STATE RELEASE TEST}

To perform this test $40 \mathrm{ml}$ of argon was injected until reaching steady state. During the injection process the same unstable period was observed due to the changes in the rheology of the simulant. Afterwards, the process of release started. Throughout the release process three zones of rate of release were detected. The first one is associated to the release of smaller bubbles, the second one to the bigger bubbles and the third one to other gases accumulated in the simulant. In the following figures 14 and 15 the rate of release can be observed. Respectively the rates of release are tabulated in table 4 . 


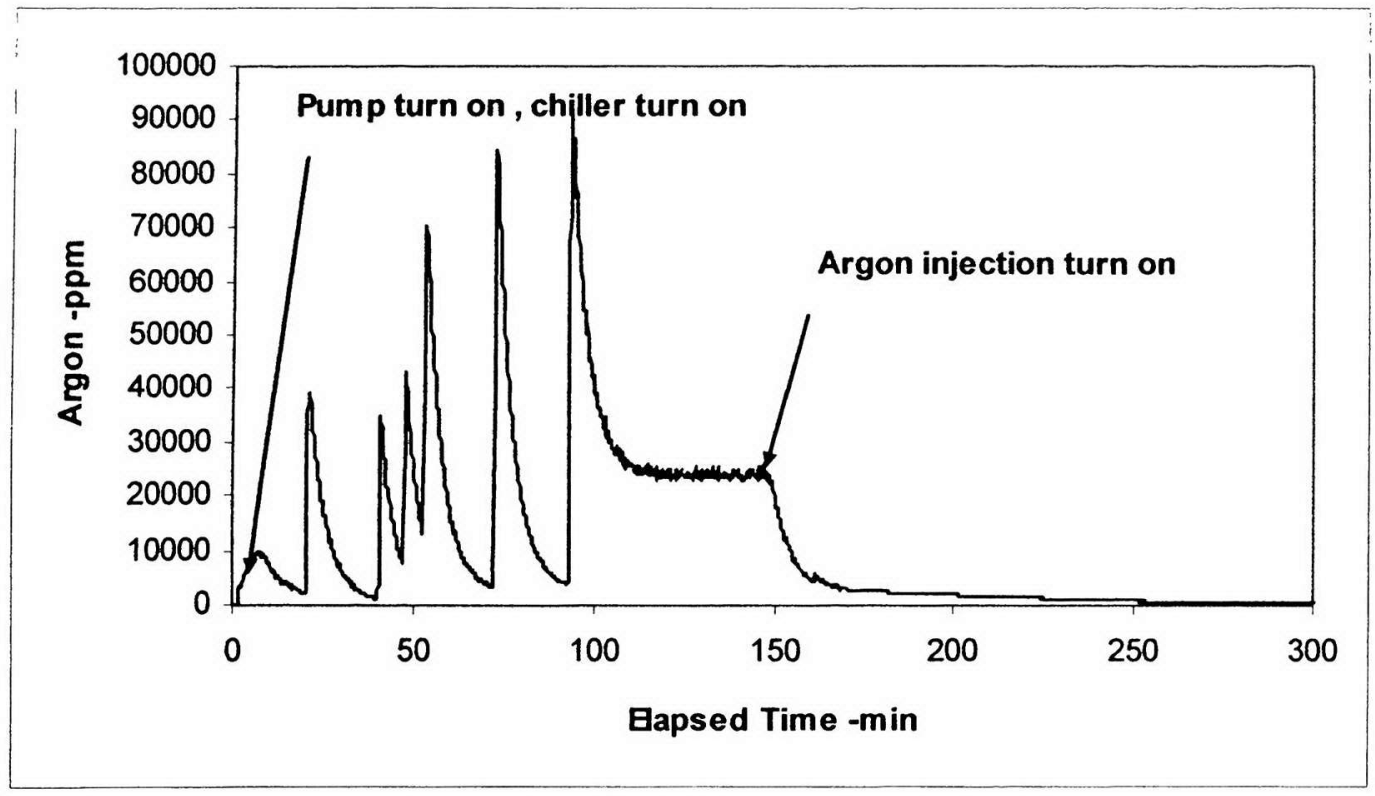

Figure 17 Argon Concentration during Release into Qard 13.5 until reaching steady state condition.

Table 5 and figure 19 show a comparison of rate of release for QARD 13.5 and clay $27 \%$. It can be noted that clay $27 \%$ which is the simulant with greater value for shear strength and viscocity has a greater rate of release compare to QARD 13.5. 


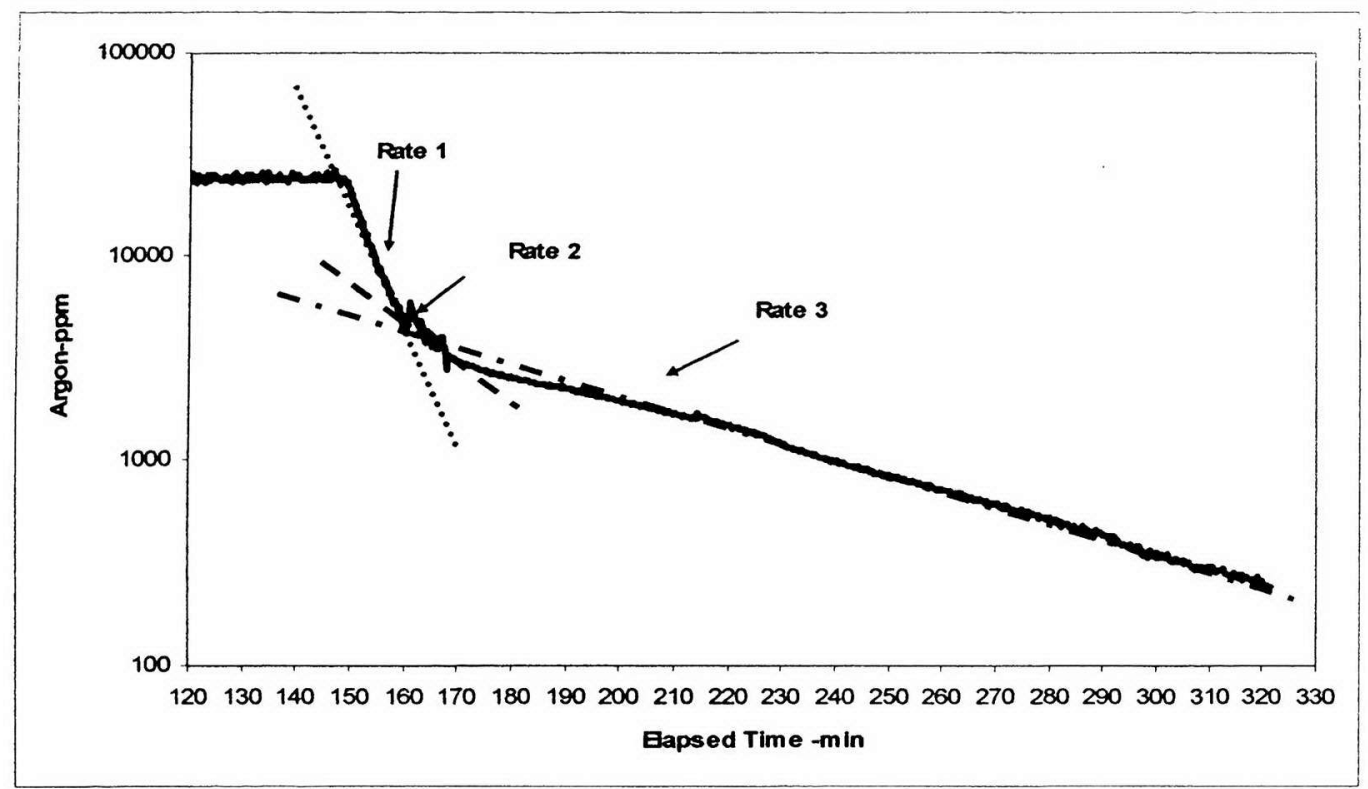

Figure 18. Rate of Release from Qard 13.5 during Steady State Release

\begin{tabular}{|c|c|c|c|c|}
\hline $\begin{array}{c}\text { Time(X1) } \\
\text { (min) }\end{array}$ & $\begin{array}{l}\text { Argon Detected } \\
\text { (Y1) (mol) }\end{array}$ & $\begin{array}{c}\text { Time (X2) } \\
(\mathrm{min})\end{array}$ & $\begin{array}{l}\text { Argon Detected } \\
\text { (Y2) (mol) }\end{array}$ & $\begin{array}{c}\text { RATE OF } \\
\text { RELEASE (R) } \\
R=(\mathrm{Y} 2-\mathrm{Y} 1) /(\mathrm{X} 2-\mathrm{X} 1) \\
(\mathrm{mol} / \mathrm{min}) \\
\end{array}$ \\
\hline 150.4 & 19686 & 154.1 & 17316.22 & -640.4811 \\
\hline 164.1 & 3649 & 166.6 & 3387.09 & -104.764 \\
\hline 183.4 & 2407.03 & 185 & 2336.23 & -44.25 \\
\hline
\end{tabular}

Table 3 Rate of Release from Qard 13.5 during Steady State Release 


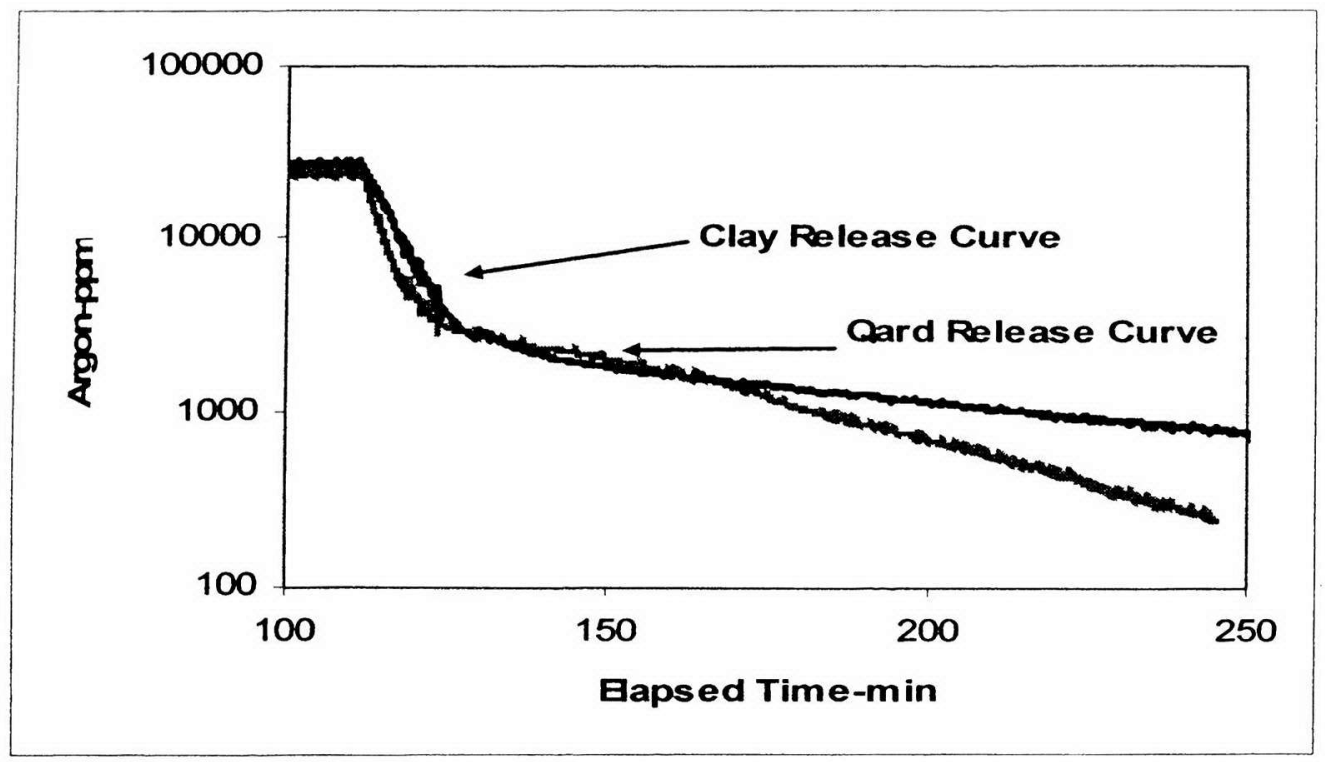

Figure 19 Rate of Release Comparison Curve for QARD and Clay 


\begin{tabular}{|c|c|c|c|c|c|c|c|}
\hline Simulant & $\begin{array}{c}\text { Shear } \\
\text { Strength } \\
(\mathrm{Pa}) \\
\end{array}$ & $\begin{array}{c}\text { Consistency } \\
\text { (cP) } \\
\end{array}$ & $\begin{array}{l}\text { TimeX1 } \\
(\min ) \\
\end{array}$ & \begin{tabular}{|c|}
$\begin{array}{c}\text { Argon } \\
\text { Release Y1 }\end{array}$ \\
(PPM) \\
\end{tabular} & $\begin{array}{r}\text { Time X2 } \\
(\mathrm{min}) \\
\end{array}$ & \begin{tabular}{|c|} 
Argon \\
ReleaseY2 \\
(PPM) \\
\end{tabular} & $\begin{array}{l}\text { Rate of } \\
\text { Release } \\
\end{array}$ \\
\hline \multicolumn{8}{|l|}{ QARD } \\
\hline \multirow[t]{3}{*}{13.5} & \multirow[t]{3}{*}{6.3} & \multirow[t]{3}{*}{17.3} & 150.4 & 19686 & 154.1 & 17316.22 & -640.48 \\
\hline & & & 164.1 & 3649 & 166.6 & 3387.09 & -104.76 \\
\hline & & & 183.4 & 2407.03 & 185 & 2336.23 & -44.25 \\
\hline \multicolumn{8}{|l|}{ Clay } \\
\hline \multirow[t]{3}{*}{$27 \%$} & \multirow[t]{3}{*}{30} & \multirow[t]{3}{*}{24} & 119.3 & 8709.31 & 123.4 & 5222.06 & -850.55 \\
\hline & & & 139.6 & 2137.45 & 141.8 & 2044.66 & -42.18 \\
\hline & & & 204 & 1164.44 & 216.3 & 1022.54 & -11.54 \\
\hline
\end{tabular}

Table 4 Comparison of Rate of Release from QARD 13.5 to Clay $27 \%$ 


\subsection{RESULTS ANALYSIS}

The results obtained from the experiments can be summarized as follows:

- QARD and Clay simulants have similar holdup behaviour despite the difference in rheology.

- The approximate percentage of holdup for both simulants was of about $3 \%$.

- Clay $27 \%$ simulant rate of release was about $75 \%$ greater than QARD

- The shear strength and consistency properties facilitate the gas release within the simulants. Simulants with greater shear strength and consistency showed a larger rate of release. 
The main objective of this work was to obtain information for comparison of gas retention and release behavior of clay based simulants to the precipitated hydroxide QARD simulant. Therefore, there were limitations of argon as surrogate to hydrogen was acknowledged, but not explored. Also, a mechanically well mixed vessel was considered to represent the bounding condition of a well mixed vessel during PJMs.

The conclusions are summarized as follows:

- Increased injection did not result in higher retention. Both Qard and the $27 \%$ Clay simulants seemed to thicken during the injection period, requiring continual adjustment to the mixer speed. These adjustments to the mixer speed were followed by a surge in the effluent argon level.

- Both the $27 \%$ Clay simulant and the Diluted Clay simulant appear to exhibit faster gas release behavior than the Qard simulant. In other words, the clay based simulants tested may be used to provide an upper bound for the gas release rate from the gelled Qard simulant.

- Even though extensively different in rheology, the $27 \%$ Clay simulant and diluted clay simulant tested exhibit similar gas release behavior within the range of initial gel holdups in these tests.

- The rate of release for $27 \%$ Clay was greater compare to Qard 13.5 due to the greater value in shear strength and consistency. 
- The steady state retention levels were generally low, on the order of about $3 \%$ by volume.

\section{$\underline{\text { Recommendations: }}$}

- Tests with hydrogen would provide a better understanding of the complexity of hydrogen release inside the waste tanks.

- Design and construct a pilot testing system of about 60 gallons that would provide a more realistic set of data.

- Geometry of the vessel should account for bubble compressibility. 


\section{References}

1. G. Terrones and Gauglitz, "Vessel - Spanning Bubble Formation in $\mathrm{K}$ - Basin Sludge Stored in Large - Diameter Container", , Pacific Northwest National Laboratory, March 2002

2. Phillips A. Gauglitz et al "Mechanics of Bubbles in Sludge and Slurries", Pacific Northwest National Laboratory, 2002

3. G.D. Johnson et al, "Flammable Gas Safety Issue Resolution", CH2M HILL Hanford Group, 2002

4. P.A. Gauglitz et al, "Mechanisms of Gas Bubble Retention", Pacific Northwest laboratory, September 1994

5. P.A. Gauglitz et al, "Gas Distribution Effects on Waste Properties: Viscosities of Bubbly Slurries", Pacific Northwest Laboratory, September 1994

6. S.D. Rassat et al, "A Discussion of SY-101 Crust Gas Retention and Release Mechanisms" Pacific Northwest National Laboratory, February 1999

7. J. Tingey et al, "Physical Property and Rheological Testing of Actual Transuranic Waste from Hanford Single - Shell Tanks", Pacific Northwest National Laboratory, August 2003

8. BE Wells et al, "The Potential for Buoyant Displacement Gas Release Events in Tank 241 - SY - 102 after Waste Transfer from Tank 241-SY-101", Pacific Northwest National Laboratory, April 2000

9. M.I.Urseanu, R.P.M. Guit, A. Stankiewicz, G. van Kranenburg, J.H.G.M. "Influence of Operating Pressure on the Gas Holdup in Bubble Columns for High Viscous Media", Chemical Engineering Science, Vol. 58, p.p. 697-704.

10. A. Colmenares, M. Sevilla, J.J. Concalves and D. Gonzalez - Mendizábal "Fluid Dynamics Experimental Study in a Bubble Column with Internals", Int. Comm. Heat Mass Transfer, vol. 28, No. 3, pp. 389 - 398, 2001.

11. Seraphim D. Vlaev, Minka D. Valeva and Reg Mann "Some Effects of Rheology on the Spatial Distribution of Gas Holdup in Mechanical Agitated Vessel", Chemical Engineering Journal, Vol. 87, p.p. 21 - 30, 2001.

12. D.J. Sherwood and L.M.Stock. "Modifying the Hu Correlation to Predict Hydrogen Formation in the Hanford Waste Treatment and Immobilization Plant", WTP Report: 24590-WTP-RPT-RT-04-0002, 2003 
13. P.Sundar, "Gas Retention and Release Tests in Non- Newtonian Simulants at Florida International University in support of the WTP Project", WTP Report: 24590-PTFRPT-RT-04-0005-Rev.0, 2004. 
APPENDIX 


\section{Appendix A}

\section{Static Mixer Calibration}

The objective of this test is to determine the suitable simulant recirculation flow rate and static mixer length that will provide gas bubbles less than $1 \mathrm{~mm}$ in diameter. The test will be carried out using Laponite simulant provided by WTP.

Test Set - Up

The equipment set-up for this test in shown in Figure 20 It consists primarily of pumping the simulant from the simulant tank through a static mixer back to the tank in a recycle loop. The tracer gas, argon, is fed to the intake of the pump. The liquid phase mixed with the entrained gas passes through the static mixer to create smaller bubbles of entrained gas. Provision is made to collect a slip-stream of the static mixer discharge for visual examination and measurement of the bubble size obtained. 


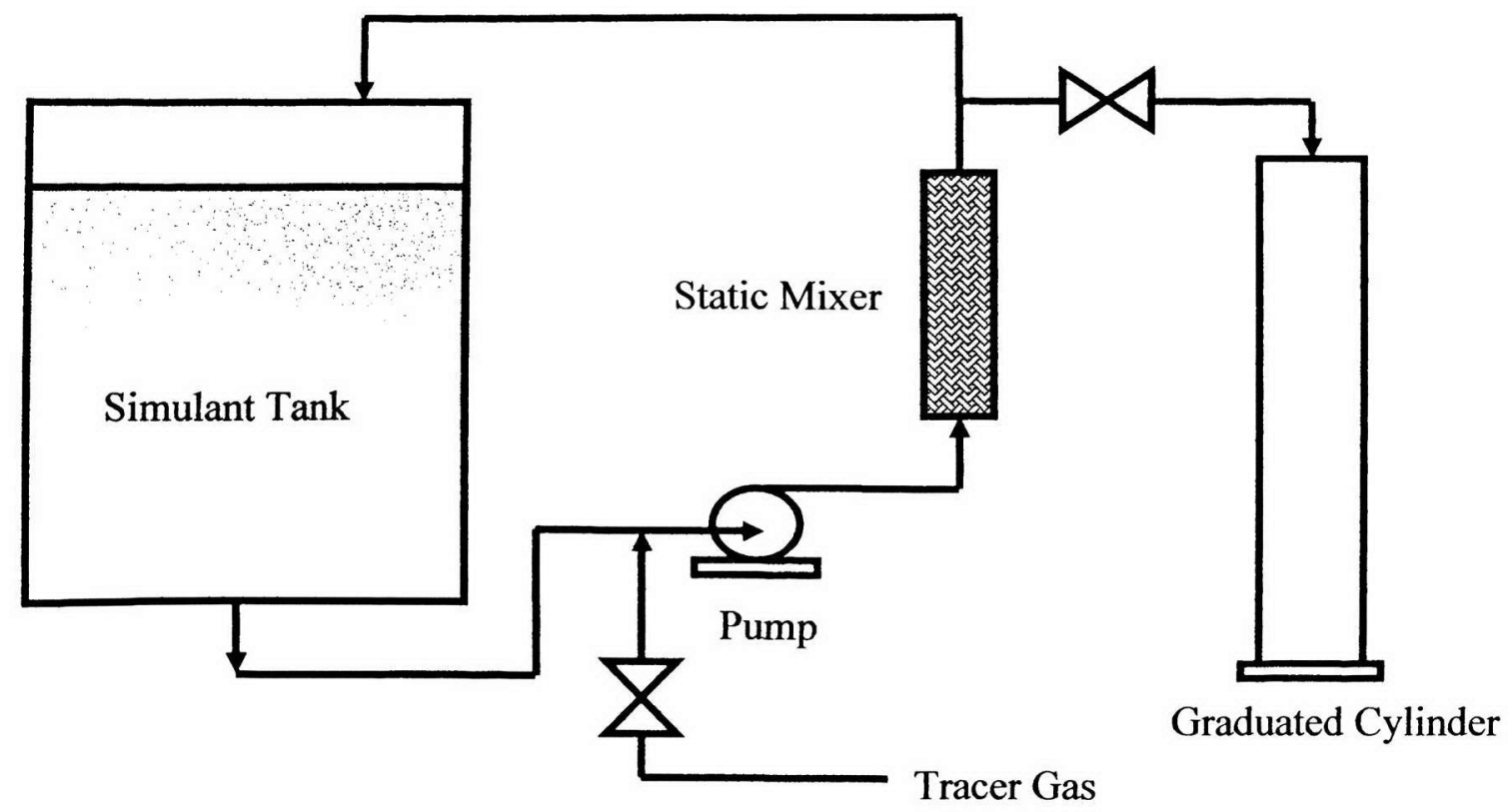

Figure 20 Schematic Test Set up for Static Mixer Scaling Test

It is important to locate the recirculation pump at an elevation sufficiently below the Laponite simulant tank to avoid pump cavitation. Past experience at WTP indicates that a $4 \mathrm{ft}$ difference in elevation between the tank and the pump intake may be adequate.

Test Procedure

It is important that the entire volume of the simulant phase has been subject to full shear before testing is started. Therefore, the procedure provides for elongated mixing and pump recirculation periods before gas injection. The test logic is illustrated in Figure 21.

The equipment set-up for this test in shown in Figure 20. It consists primarily of pumping the simulant from the simulant tank through a static mixer back to the tank in a 
recycle loop. The tracer gas, argon, is fed to the intake of the pump. The liquid phase mixed with the entrained gas passes through the static mixer to create smaller bubbles of entrained gas. Provision is made to collect a slip-stream of the static mixer discharge for visual examination and measurement of the bubble size obtained.

- Start the mixer and gradually increase mixer speed until well defined movement of the simulant is observed over the entire top surface as well as at the walls. Note and record the mixer speed. Increase the mixer speed to $20 \%$ over this speed and continue to mix the simulant for at least 30 minutes, and preferably 1 hour.

- Start the recirculation pump at the end of the mixing period and recirculate through the static mixer at a fixed rate prescribed for the test for 30 minutes to 1 hour.

- Introduce gas injection for 30 secs. Capture a sample of the liquid discharge from the static mixer in a small diameter, tall container (such as a graduated cylinder) to allow visual examination of the bubble size.

- Depending on the bubble size observed take the action as indicated in the Figure 21 . Note that increase in pump flow rate is to be made per test plan in Table 5.

- Record pump flow rate and static mixer dimensions (or number of modules), gas injection rate and the bubble size observed. 
Table 5 Conditions for the Static Mixer Scaling Test

\begin{tabular}{|c|c|c|}
\hline Run No. & Argon / Air Flow Rate, & $\begin{array}{l}\text { Recirculation Pump, Flow } \\
\text { Rate, GPM }\end{array}$ \\
\hline 1 & 50 & 1.0 \\
\hline 2 & 50 & 5.0 \\
\hline 3 & 50 & 1.0 \\
\hline 4 & 100 & 2.5 \\
\hline 5 & 100 & 5.0 \\
\hline 6 & 100 & 1.0 \\
\hline 7 & 150 & 2.5 \\
\hline 8 & 150 & 5.0 \\
\hline 9 & 150 & \\
\hline
\end{tabular}

This test will seek to identify at least one operating point for the recirculation pump rate (GPM) and static mixer length (or configuration) that will result in gas bubbles with a maximum diameter of about $1 \mathrm{~mm}$ using Laponite and a gas injection rate of 50 $\mathrm{ml} / \mathrm{min}$. It is preferable to have a mean gas bubble diameter less than $0.5 \mathrm{~mm}$. This test does not require the fully assembled system and can be accomplished off-line using an open vessel ( 4 liter beaker) in the place of the test vessel.

The objective of this test is to determine the suitable simulant flow rate and static mixer length that will provide gas bubbles less than $1 \mathrm{~mm}$ in diameter. The test will be carried using Laponite simulant provided by WTP. 


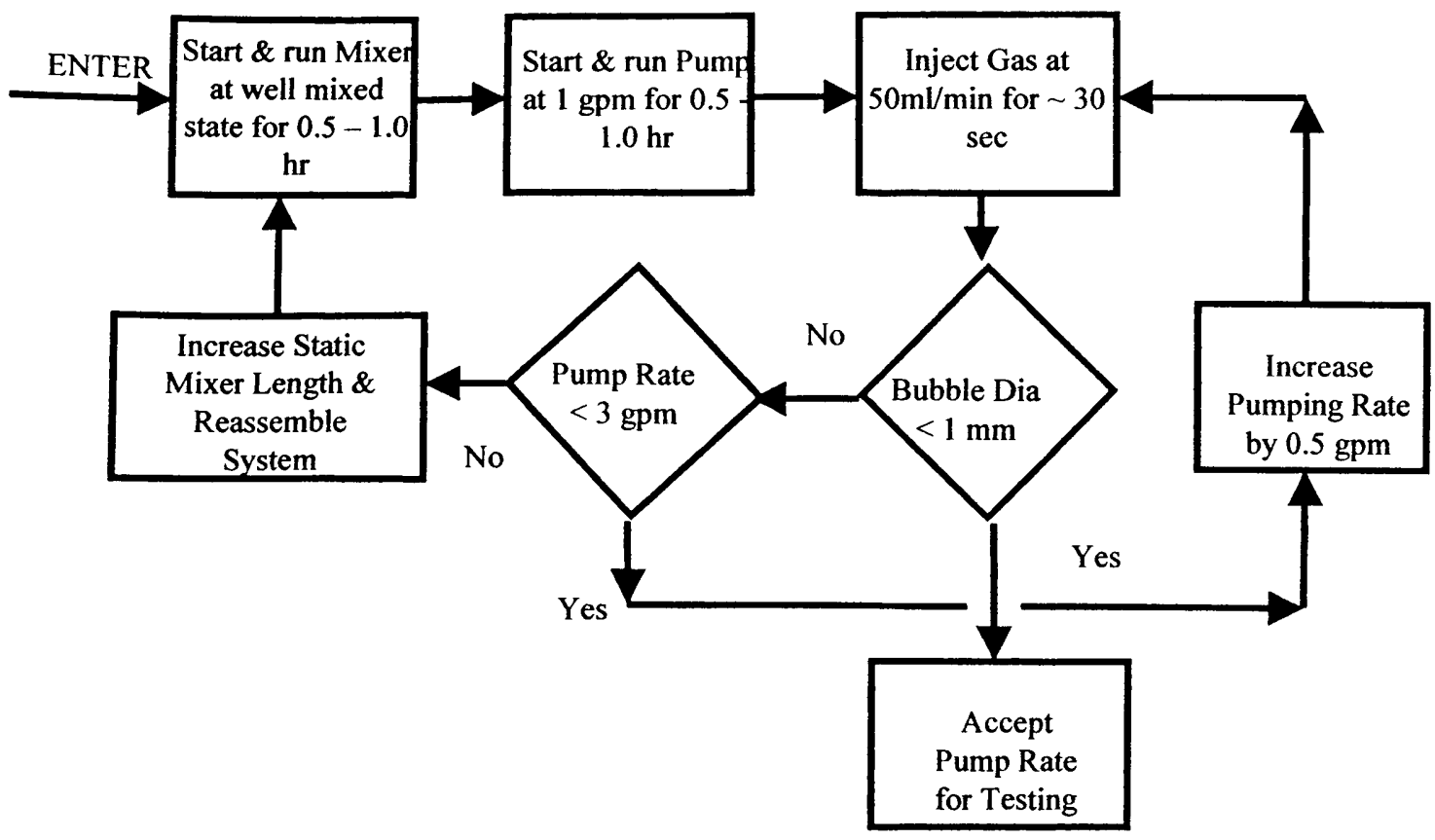

Figure 21 Logic Diagram for Static Mixer Scaling Test 
Before commencement of any gas retention and release testing, it is paramount that the simulant is in the fully mixed condition. The following procedure is to be followed for each of the simulants to determine the state of mixing before gas addition:

- Add a 5 gallon charge of the simulant to the test vessel making sure that all the valves leading to and out of the vessel are closed.

- Start the mixer and increase the speed very gradually without cavitation or cavern formation. Increase the mixer speed until there is visible movement of liquid over the entire top surface and the wall area. If necessary, use flow beads as tracers to verify this. Continue mixing the simulant for at least 30 minutes.

- Examine visually to ensure no stagnant zones develop during the 30 minute mixing period. If examination indicates no stagnant areas, note the mixer speed specific to the simulant being tested. The actual mixer speed to be used during gas retention and release tests will be $20 \%$ above the mixer speed noted. 


\section{Appendix B}

Gas Properties:

Hydrogen

Henry's Law constant (water solution)

Table 6 Henry's law constant $\left(\mathrm{k}_{\mathrm{H}}^{\mathrm{O}}\right)$ for solubility in water at $298.15 \mathrm{~K}(\mathrm{~mol} / \mathrm{kg}$ *bar)

\begin{tabular}{|r|r|l|l|}
\hline $\mathbf{k}^{\circ}{ }_{\mathrm{H}}\left(\mathrm{mol} / \mathrm{kg}^{*}\right.$ bar $)$ & $\mathrm{d}\left(\ln \left(\mathrm{k}_{\mathrm{H}}\right)\right) / \mathrm{d}(\mathrm{l} / \mathrm{T})(\mathrm{K})$ & Method & \multicolumn{1}{|c|}{ Reference } \\
\hline 0.00078 & 500. & $\underline{L}$ & Lide and Frederikse, 1995 \\
\hline 0.00078 & 640. & $\mathrm{Q}$ & $\underline{\text { Dean, 1992 }}{ }^{3}$ \\
\hline 0.00078 & 490. & $\underline{\text { Lilhelm, Battino, et al., 1977 }}$ \\
\hline 0.00078 & & $\underline{\mathrm{R}}$ & $\underline{\text { Hine and Weimar, 1965 }}$ \\
\hline
\end{tabular}

${ }^{3}$ Only the tabulated data between $\mathrm{T}=273 . \mathrm{K}$ and $\mathrm{T}=303 . \mathrm{K}$ from Dean, 1992 was used to derive $k_{H}$ and $-\Delta k_{H} / R$. Above $T=303$. $K$ the tabulated data could not be parameterized by equation (reference missing) very well. The partial pressure of water vapor (needed to convert some Henry's law constants) was calculated using the formula given by Sander, Lelieveld, et al., 1994. The quantities A and $\alpha$ from Dean, 1992 were assumed to be identical. 
Nitrogen

Table 7 Henry's law constant $\left(\mathrm{k}_{\mathrm{H}}^{\circ}\right)$ for solubility in water at $298.15 \mathrm{~K}(\mathrm{~mol} / \mathrm{kg}$ *bar $)$

\begin{tabular}{|r|r|c|l|}
\hline $\mathrm{k}^{\circ}{ }_{\mathrm{H}}\left(\mathrm{mol} / \mathrm{kg}^{*}\right.$ bar $)$ & $\mathrm{d}\left(\ln \left(\mathrm{k}_{\mathrm{H}}\right)\right) / \mathrm{d}(1 / \mathrm{T})(\mathrm{K})$ & Method & \multicolumn{1}{|c|}{ Reference } \\
\hline 0.00060 & 1300. & $\underline{\mathrm{X}}$ & $\underline{\text { Kavanaugh and Trussell, 1980 }}$ \\
\hline 0.00065 & 1300. & $\underline{\mathrm{L}}$ & Wilhelm, Battino, et al., 1977 \\
\hline
\end{tabular}

Argon

Table 8 Henry's law constant $\left(\mathrm{k}_{\mathrm{H}}^{\circ}\right)$ for solubility in water at $298.15 \mathrm{~K}\left(\mathrm{~mol} / \mathrm{kg}^{*}\right.$ bar $)$

\begin{tabular}{|r|r|c|l|}
\hline $\mathrm{k}_{\mathrm{H}}^{\mathrm{O}}\left(\mathrm{mol} / \mathrm{kg}^{*}\right.$ bar $)$ & $\mathrm{d}\left(\ln \left(\mathrm{k}_{\mathrm{H}}\right)\right) / \mathrm{d}(1 / \mathrm{T})(\mathrm{K})$ & Method & Reference \\
\hline 0.0014 & 1500. & $\underline{\mathrm{L}}$ & Wilhelm, Battino, et al., 1977 \\
\hline 0.0014 & 1100. & $\underline{\mathrm{M}}$ & Morrison and Johnstone, 1954 \\
\hline
\end{tabular}

Method:

C The paper that is cited here refers to another reference which could not be obtained (e.g., personal communication, Ph.D. theses, internal papers etc.).

E The value is estimated. Estimates are only listed if no reliable measurements are available for the compound.

$\mathrm{L}$ The cited paper is a literature review.

M Original publication of a measured value. 
$\mathrm{R}$ The cited paper presents a recalculation of previously published material (e.g., extrapolation to a different temperature or concentration range).

$\mathrm{T}$ Thermodynamical calculation.

V Vapor pressure of the pure substance is used to determine the Henry's law constant.

$\mathrm{X}$ The information presented comes from another paper or personal communication.

Q The cited paper doesn't clearly state how the value was obtained. 


\section{Appendix C}

Simulants

Table 9 Blue Laponite Shipping Details and Properties

\begin{tabular}{|l|l|}
\hline \multicolumn{1}{|c|}{ Shipment Details: } & \\
\hline Airbill & $\# 032229057$ \\
\hline Date Shipped on: & $11 / 18 / 2003$ \\
\hline Total Weight & $190 \mathrm{lbs}$ \\
\hline \multicolumn{1}{|c|}{ Simulant Properties } & \\
\hline Initial Shear strength & $100 \mathrm{~Pa}$ \\
\hline \multicolumn{1}{|c|}{ Laponite after Dilution } \\
& \\
\hline Calculated Final Shear strength & \\
\hline Initial mass of laponite & $100 \mathrm{mass}$ \\
\hline Mass of water added & $10.8 \mathrm{mass}$ \\
\hline Weight of Laponite & $24.06 \mathrm{~kg}$ \\
\hline Weight of Water added & $5.2732 \mathrm{~kg}$ \\
\hline Weight of Laponite Obtained & $29.355 \mathrm{~kg}$ \\
\hline
\end{tabular}

${ }^{4}$ Note: Dilution details can be found on Page 30 from the log book 
Table 10 Clay Simulant 1 (27\% Kaolin/Bentonite Slurry in Water) Shipping Details and Properties

\begin{tabular}{|l|l|}
\hline \multicolumn{1}{|c|}{ Container $\mathbf{~}^{5}$} & 5 Gallon Plastic pail \\
\hline \multicolumn{1}{|c|}{ Shipment Details } & \\
\hline Tracking \# & 601506662180 \\
\hline Date Shipped on: & $11 / 24 / 2003$ \\
\hline Container Id \# & $112303 \mathrm{~B} 3$ \\
\hline Weight: & $42 \mathrm{lbs}$ \\
\hline & \\
\hline Yield stress Container 26,7 & \\
\hline Consistency & $29.6 \mathrm{~Pa}$ \\
\hline & $24.1 \mathrm{cP}$ \\
\hline & \\
\hline Container Id \# & $5 \mathrm{Gallon}$ Plastic pail \\
\hline Tracking \# & $112303 \mathrm{~B} 1$ \\
\hline Date Shipped on: & 601506662168 \\
\hline Weight : & $11 / 24 / 2003$ \\
\hline & $40 \mathrm{lbs}$ \\
\hline Properties & \\
\hline Yield stress & \\
\hline Consistency & $30.4 \mathrm{~Pa}$ \\
\hline
\end{tabular}

${ }^{5}$ Note: Completely used

${ }^{6}$ Note: Half Used

${ }^{7}$ Note: Samples collected from this container 
Table 11 24\% Clay Simulant 2 (Kaolin / Bentonite Slurry in Water - Diluted Clay).

Shipment Details and Properties

\begin{tabular}{|c|c|}
\hline Shipment Details: & \\
\hline Container ${ }^{8}$ : & 5 Gallon Cubitainer \\
\hline Tracking \# & 601506662845 \\
\hline Date Shipped on: & $12 / 3 / 2003$ \\
\hline Weight : & $57 \mathrm{lbs}$ \\
\hline Properties & \\
\hline Yield stress & $6.7 \mathrm{~Pa}$ \\
\hline Consistency & $16.4 \mathrm{cP}$ \\
\hline
\end{tabular}

${ }^{8}$ Note: Completely Used 
Table 12 QARD Sludge Simulant ( FIU QARD Batch One), Shipment Details and Properties

\begin{tabular}{|l|l|}
\hline \multicolumn{1}{|c|}{ Shipment Details: } & \\
\hline Container: & 10 bottles (2L each) \\
\hline Tracking \# & 843659738040 \\
\hline Date Shipped on: & $11 / 17 / 2003$ \\
\hline \multicolumn{1}{|c|}{ Properties Rheology at $25^{\circ} \mathrm{C}$} & \\
\hline Yield stress & \\
\hline Consistency & $6.3 \mathrm{~Pa}$ \\
\hline pH & $17.3 \mathrm{cP}$ \\
\hline & 13.5 \\
\hline Properties Rheology at $40^{\circ} \mathrm{C}$ & \\
\hline Yield stress & $6.5 \mathrm{~Pa}$ \\
\hline
\end{tabular}




\section{Appendix D}

Calibration Results for Residual Gas Analyzer (RGA)

Calibration for the RGA was performed, from Hidden Analytical Instruments (HA-085-005) on November 22, 2003 in the Slurry lab (CEAS 2110). The pressure recorded on the first and second vacuum stages are $2.0 \times 10^{-4}$ torr and $1.6 \times 10^{-6}$ torr. Before the calibration, the pump was allowed to run continuously for 4 days to get it stabilized.

Calibration Standards

Calibration gases were purchased from Air Gas Instruments $\left(9030\right.$ N.W. $58^{\text {th }}$ Street. Miami, FL 33178).

Argon

Table 13. Calibration Standards and Certificate of Conformance for Argon

\begin{tabular}{|l|l|}
\hline \multicolumn{1}{|c|}{ Concentration Ar/ $\mathrm{N}_{2}$} & \multicolumn{1}{c|}{ Certificate of Conformance } \\
\hline 0 & 24590 -WTP-CGD-RT-03-001 \\
\hline 84.2 & 24590 -WTP-CGD-RT-03-001 \\
\hline 1501 & 24590 -WTP-CGD-RT-03-001 \\
\hline 5013 & 24590 -WTP-CGD-RT-03-001 \\
\hline 99600 & 24590 -WTP-CGD-RT-03-001 \\
\hline
\end{tabular}


Argon Calibration

Argon Concentration $=0 \mathrm{ppm}$

This graph shows that the detection limit of Argon in Nitrogen is expected around $1 \mathrm{x}$ E$05 \mathrm{ppm}$.

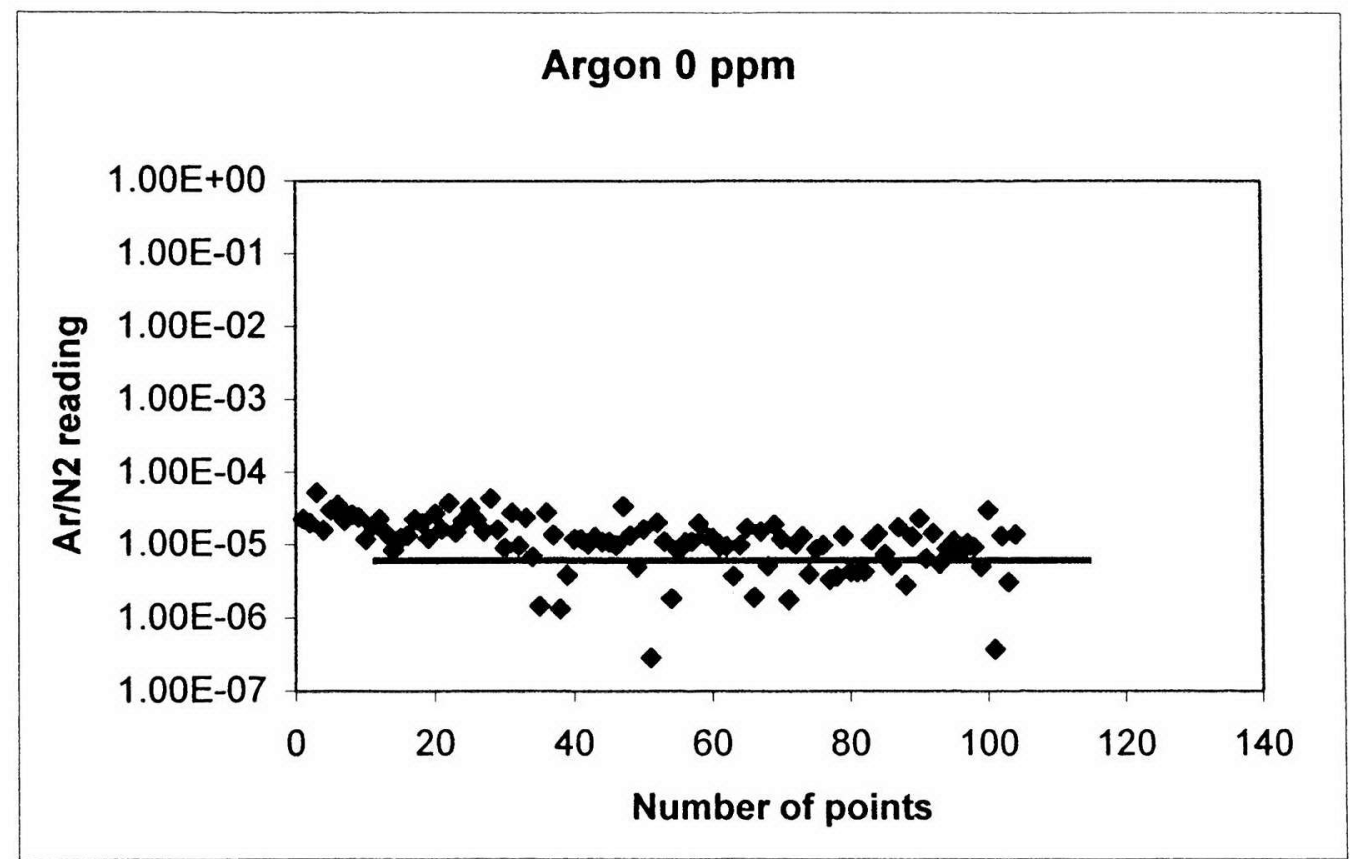

Figure 22 Calibration Graph for Argon Concentration $=0 \mathrm{ppm}$ 
Table 14 Statistical Value for Argon Concentration $=0 \mathrm{ppm}$

\begin{tabular}{|c|c|}
\hline Mean & $1.372 \mathrm{E}-05$ \\
\hline Standard Error & $9.1999 \mathrm{E}-07$ \\
\hline Median & $1.19494 \mathrm{E}-05$ \\
\hline Standard Deviation & $9.38209 \mathrm{E}-06$ \\
\hline Sample Variance & $8.80236 \mathrm{E}-11$ \\
\hline Kurtosis & 2.481131935 \\
\hline Skewness & 1.3084904 \\
\hline Range & $5.15789 \mathrm{E}-05$ \\
\hline Minimum & $2.80245 \mathrm{E}-07$ \\
\hline Maximum & $5.18591 \mathrm{E}-05$ \\
\hline Sum & 0.001426879 \\
\hline Count & 104 \\
\hline Confidence Level(95.0\%) & $1.82458 \mathrm{E}-06$ \\
\hline
\end{tabular}


Argon Concentration $=84.2 \mathrm{ppm}$

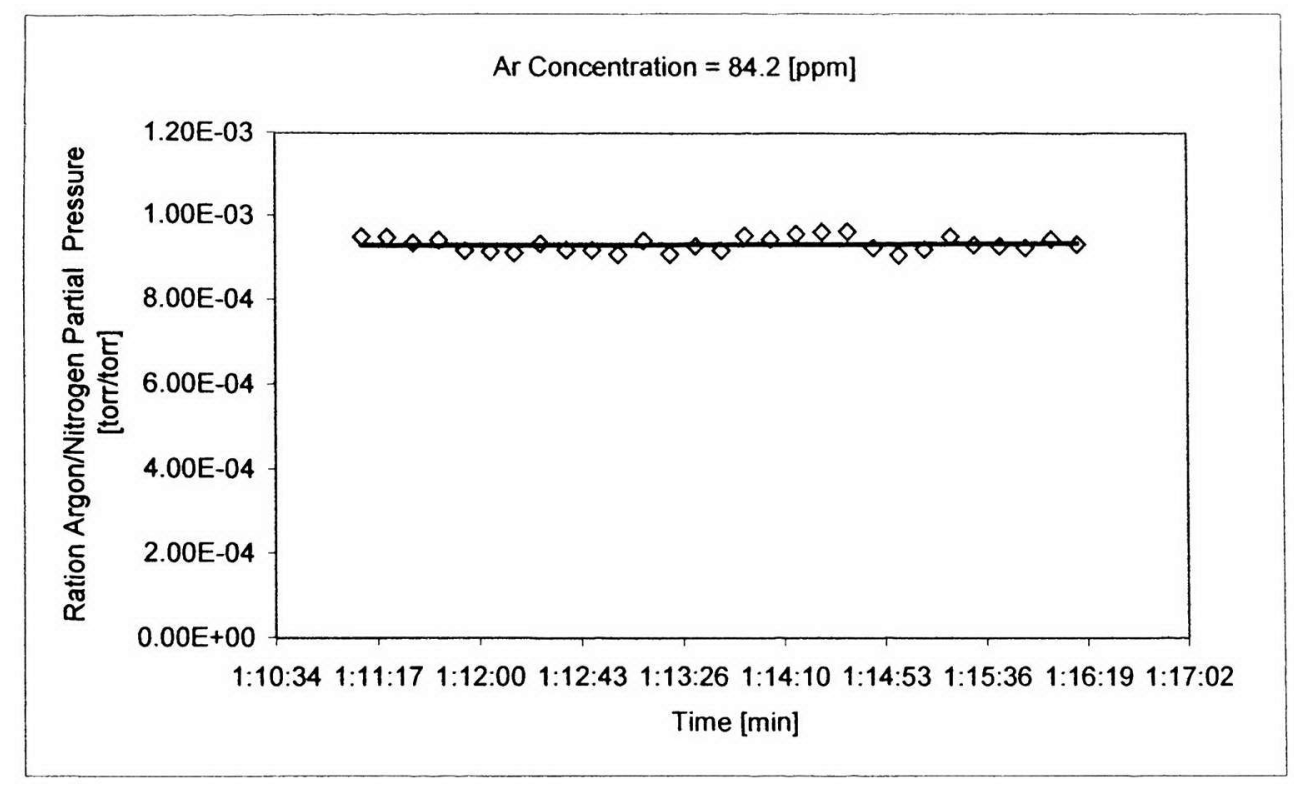

Figure 23 Calibration Graph for Argon Concentration $=82.4 \mathrm{ppm}$ 
Table 15 Statistical Value for Argon Concentration $=84.2 \mathrm{ppm}$

\begin{tabular}{|c|c|}
\hline Mean & 0.000933 \\
\hline Standard Error & $3.13 \mathrm{E}-06$ \\
\hline Median & 0.000932 \\
\hline Standard Deviation & $1.69 \mathrm{E}-05$ \\
\hline Sample Variance & $2.85 \mathrm{E}-10$ \\
\hline Kurtosis & -1.00665 \\
\hline Skewness & 0.278757 \\
\hline Range & $5.62 \mathrm{E}-05$ \\
\hline Minimum & 0.000909 \\
\hline Maximum & 0.000965 \\
\hline Sum & 0.027068 \\
\hline Count & 29 \\
\hline Largest(1) & 0.000965 \\
\hline Smallest(1) & 0.000909 \\
\hline Confidence Level(95.0\%) & $6.42 \mathrm{E}-06$ \\
\hline
\end{tabular}


Argon Concentration $=1501 \mathrm{ppm}$

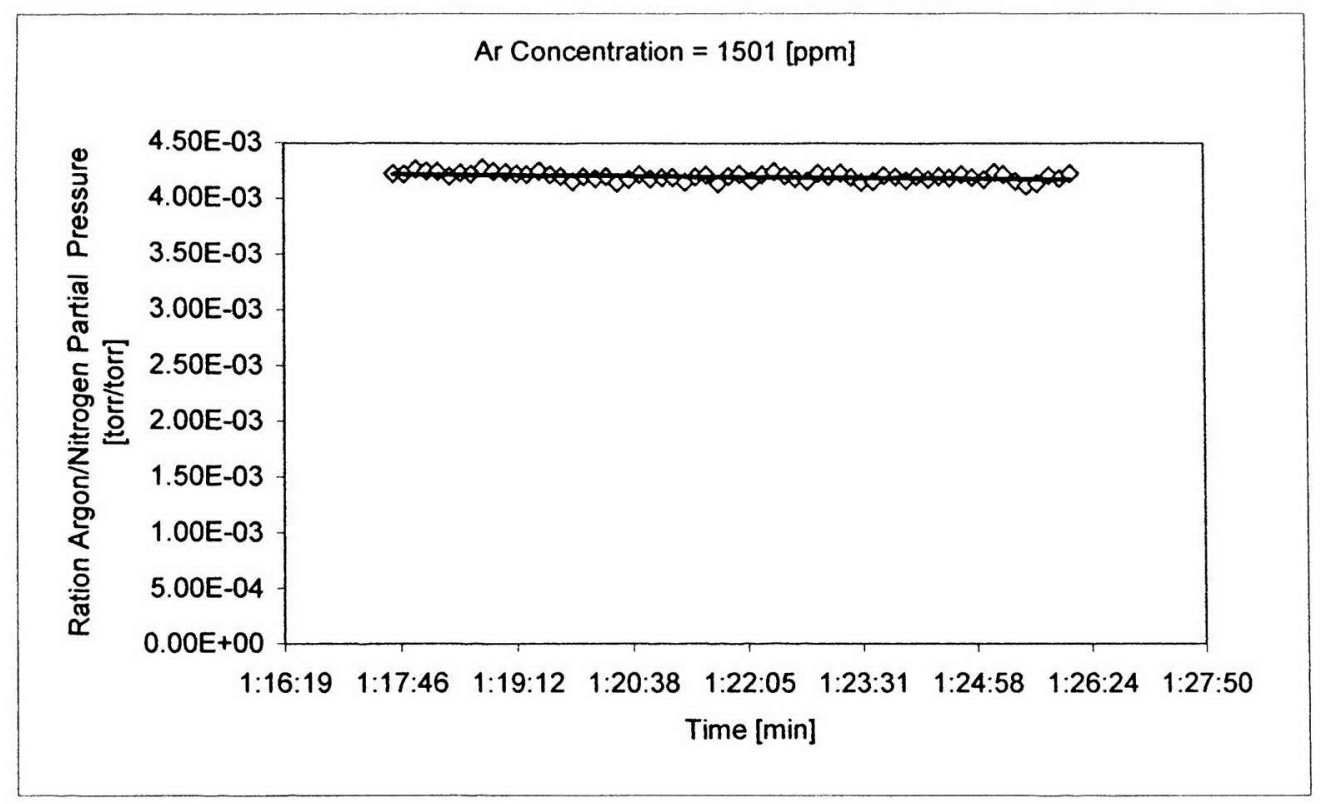

Figure 24 Calibration Graph for Argon Concentration $=1501$ ppm 
Table 16 Statistical Value for Argon Concentration $=1501 \mathrm{ppm}$

\begin{tabular}{|c|c|}
\hline Mean & 0.0042 \\
\hline Standard Error & 4.27E-06 \\
\hline Median & 0.004202 \\
\hline Standard Deviation & $3.36 \mathrm{E}-05$ \\
\hline Sample Variance & $1.13 \mathrm{E}-09$ \\
\hline Kurtosis & -0.22974 \\
\hline Skewness & -0.24367 \\
\hline Range & 0.000159 \\
\hline Minimum & 0.004117 \\
\hline Maximum & 0.004276 \\
\hline Sum & 0.260416 \\
\hline Count & 62 \\
\hline Largest(1) & 0.004276 \\
\hline Smallest(1) & 0.004117 \\
\hline Confidence Level $(95.0 \%)$ & $8.54 \mathrm{E}-06$ \\
\hline
\end{tabular}




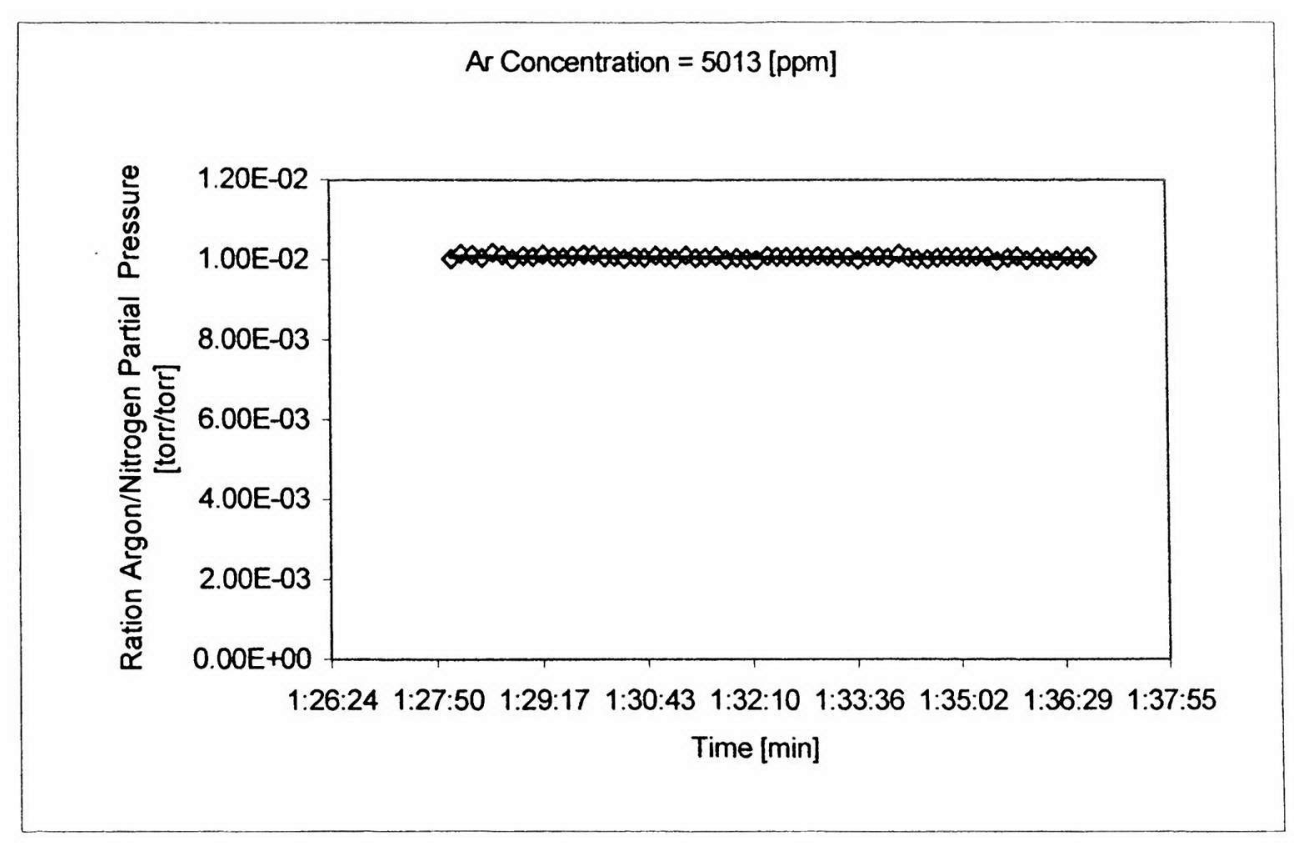

Figure 25 Calibration Graph for Argon Concentration $=5013 \mathrm{ppm}$ 
Table 17 Statistical Value for Argon Concentration $=5013 \mathrm{ppm}$

\begin{tabular}{|c|c|}
\hline Mean & 0.010058 \\
\hline Standard Error & $5.31 \mathrm{E}-06$ \\
\hline Median & 0.010062 \\
\hline Standard Deviation & $4.25 \mathrm{E}-05$ \\
\hline Sample Variance & $1.81 \mathrm{E}-09$ \\
\hline Kurtosis & 0.062748 \\
\hline Skewness & 0.032017 \\
\hline Range & 0.000206 \\
\hline Minimum & 0.009962 \\
\hline Maximum & 0.010167 \\
\hline Sum & 0.643719 \\
\hline Count & 64 \\
\hline Largest(1) & 0.010167 \\
\hline Smallest(1) & 0.009962 \\
\hline Confidence Level(95.0\%) & $1.06 \mathrm{E}-05$ \\
\hline
\end{tabular}




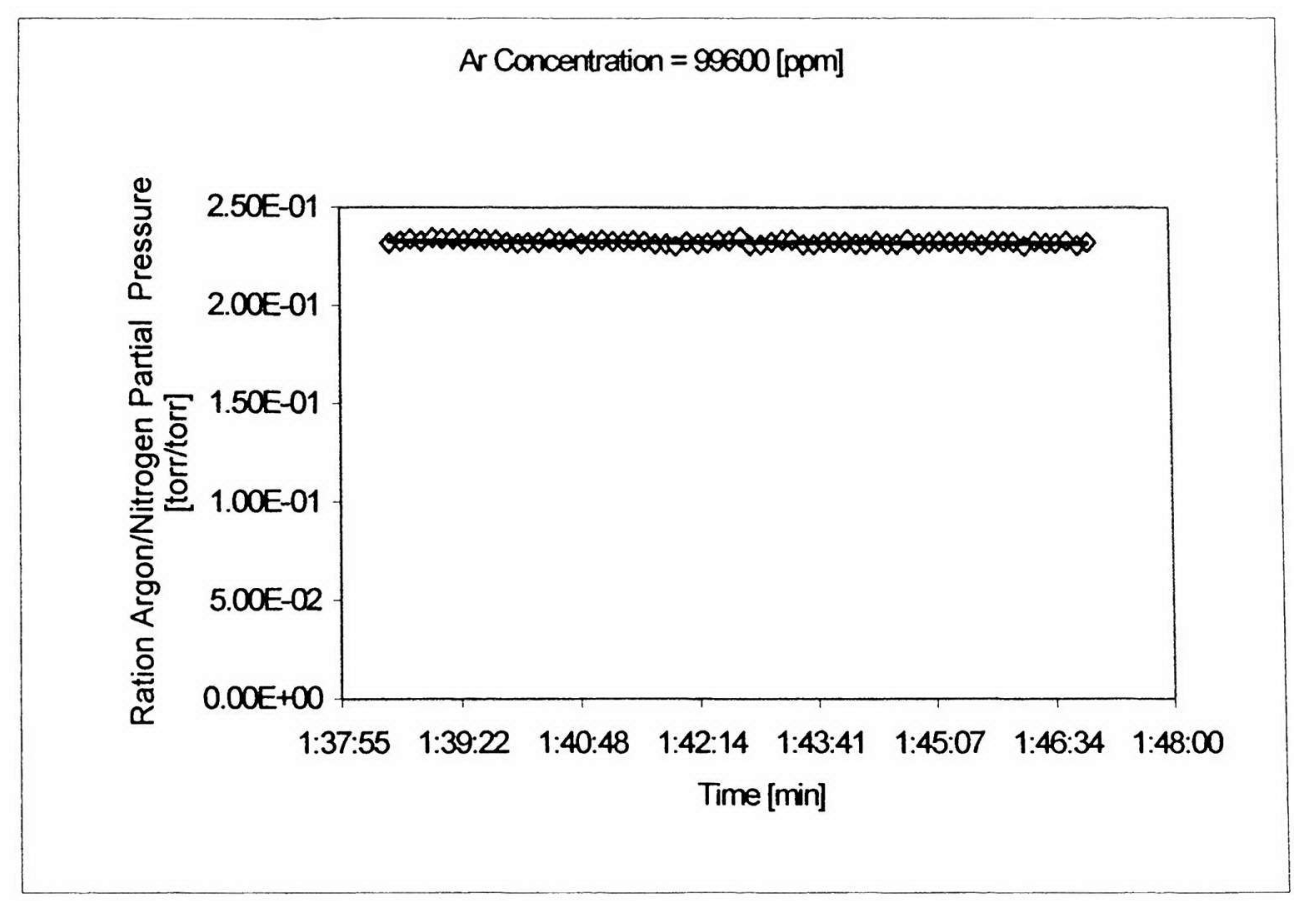

Figure 26 Calibration Graph for Argon Concentration $=99600$ ppm 
Table 18 Statistical Value for Argon Concentration $=99600 \mathrm{ppm}$

\begin{tabular}{|l|l|}
\hline Mean & 0.232204 \\
\hline Standard Error & 0.000123 \\
\hline Median & 0.232197 \\
\hline Mode & 0.231058 \\
\hline Standard Deviation & 0.001008 \\
\hline Sample Variance & $1.02 \mathrm{E}-06$ \\
\hline Kurtosis & -0.37508 \\
\hline Skewness & 0.041273 \\
\hline Range & 0.00445 \\
\hline Minimum & 0.230039 \\
\hline Maximum & 0.234489 \\
\hline Sum & 15.55768 \\
\hline Count & 67 \\
\hline Largest(1) & 0.234489 \\
\hline Smallest(1) & 0.230039 \\
\hline & 0.000246 \\
\hline & \\
\hline
\end{tabular}

Calibration curve was plotted between Argon concentration in the calibration gases and Argon/Nitrogen ratio value. The graph was plotted with error bars to include the standard deviation value associated with each point. 


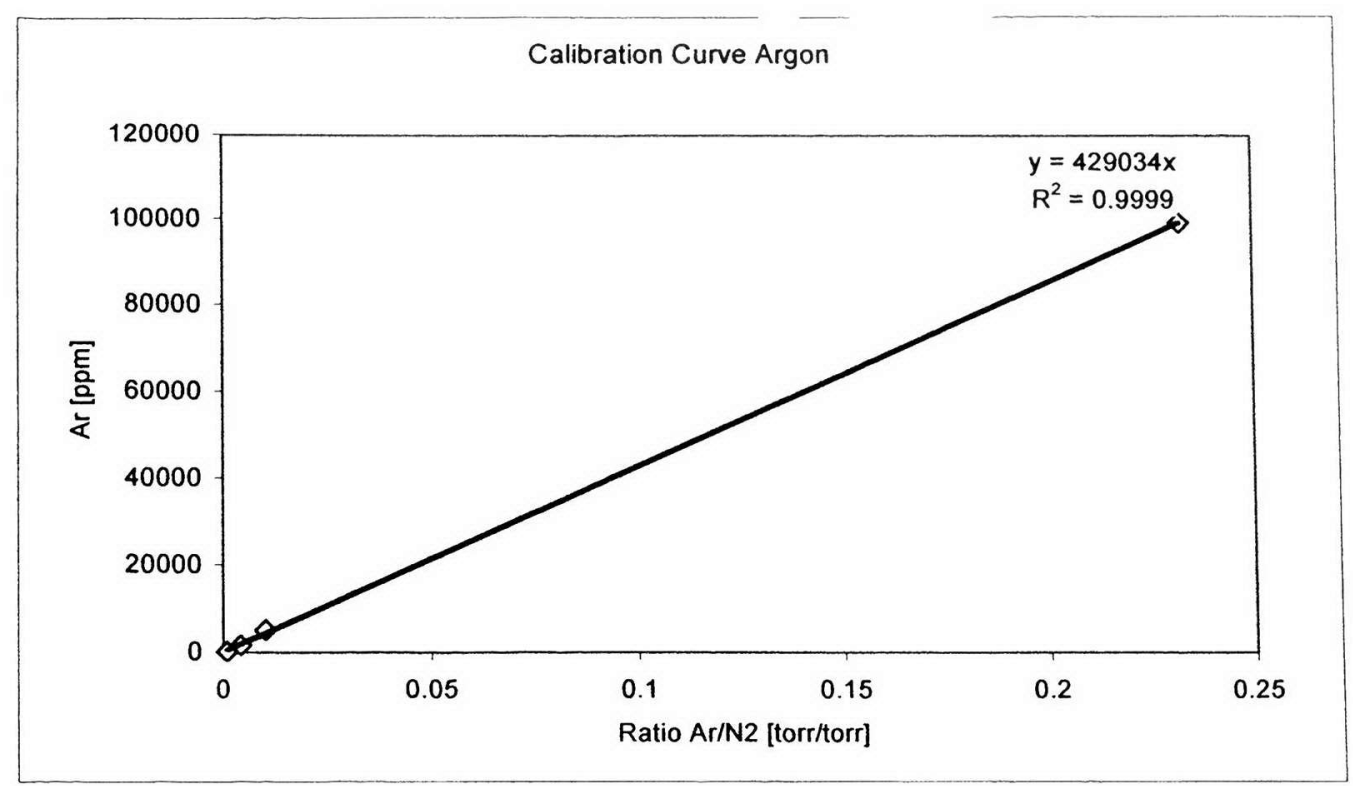

Figure 27 Calibration Curve for Argon; Concentration Range from 84.2 to 99600 ppm. 


\section{Appendix E}

Equipment Specification

Flow Meter

Manufacturer by: Brooks Instrument. Model: $5850 \mathrm{E}$

Standard Ranges:

$3 \mathrm{sccm}$ to $30 \mathrm{slpm}$

Accuracy:

$\pm 1 \%$ full scale including linearity at calibration conditions.

$\pm 1.5 \%$ full scale including linearity for flow ranges greater than $20 \mathrm{slpm}$.

Repeatability:

$0.25 \%$ of rate

Response Time:

Less than 3 seconds response to within $2 \%$ of full scale final value with a 0 to $100 \%$ command step

Power Requirements (NC Valve): $\quad+15 \mathrm{Vdc} \pm 5 \%, 35 \mathrm{~mA}$

$-15 \mathrm{Vdc} \pm 5 \%, 180 \mathrm{~mA}$

3.5 watts power consumption

Power Requirements (NO Valve): $\quad+15 \mathrm{Vdc} \pm 5 \%, 215 \mathrm{~mA}$

$-15 \mathrm{Vdc} \pm 5 \%, 180 \mathrm{~mA}$

11.85 watts power consumption 
Output Signal:

5 Volt Reference Output:

Temperature Sensitivity:

Power Supply Sensitivity:

Mounting Attitude Sensitivity:

Command Input:

Leak Integrity:

Control Range:

Mechanical Connection:

Electrical Connection:
Non-operating: $-13^{\circ} \mathrm{F}$ to $+212^{\circ} \mathrm{F}\left(-25^{\circ} \mathrm{C}\right.$ to $\left.100^{\circ} \mathrm{C}\right)$

0 - 5 Vdc into 2000 ohms or greater. Maximum ripple $3 \mathrm{mV}$

$0-5$ Volts $\pm 0.2 \%$ Maximum load $1 \mathrm{k}$ ohms

Zero: less than $\pm 0.075 \%$ F.S. per degree C.

Span: less than $\pm 1.0 \%$ F.S. shift over $10-50^{\circ} \mathrm{C}$ range

$\pm 0.09 \%$ full scale per $\%$ power supply voltage variation

$\pm 0.5 \%$ maximum full scale deviation after rezeroing

0-5 Vdc. Input resistance

$1 \times 10^{-9}$ Atm. cc/sec Helium

50 to 1

Compatible with most popular mass flow controller Card edge, 20 terminals, gold over low stress nickel plated cooper. 
Gas Analysis Systems

Manufacturer: Hyden Analytical. Model: HPR-70 Batch Inlet Gas Analysis System

- Compact bench-top / mobile cart / console rack construction.

- High Sensitivity direct inlet (to $5 \mathrm{ppb}$ ).

- Mass range options to $2500 \mathrm{amu}$.

- Liquid $\mathrm{N}_{2}$ cryopanels for optimum vacuum / minimal spectral interference.

- $1 \mathrm{~cm}^{3}$ cascade expansion to $10 \mathrm{~cm}^{3}$ to 1 litre volume.

- Direct molecular leak sampling of expanded volume.

- Minimal sample loss / zero venting / zero memory effects.

- Stability (less than $\pm 0.5 \%$ height variation over $24 \mathrm{~h}$ ).

- MASsoft control via RS232, RS485 or Ethernet LAN. 